\title{
Design of thin micro-architectured panels with extension-bending coupling effects using topology optimization
}

\author{
Filippo Agnelli ${ }^{\mathrm{a}}$, Grigor Nika ${ }^{\mathrm{b}}$, Andrei Constantinescu ${ }^{\mathrm{a}, *}$ \\ ${ }^{a}$ Laboratoire de Mécanique des Solides, CNRS, École polytechnique, Institut polytechnique de Paris, 91128 Palaiseau, France \\ ${ }^{b}$ Weierstrass Institute for Applied Analysis and Stochastics, Mohrenstrasse 39, 10117 Berlin, Germany
}

\begin{abstract}
We design the micro-architecture of thin elastic panels to control their macroscopic behavior, accounting simultaneously for in-plane stiffness, out-of-plane stiffness and the extension-bending coupling effects. Our topology optimization method combines inverse homogenization, the Hadamard shape derivative and a level set method in the diffuse interface context to systematically capture within the unit cell the optimal micro-architecture. The efficiency of the solution method is illustrated through four numerical examples where the designed shape yields an important extension-bending coupling. The deformation responses under tensile loading is assessed numerically both on the complete periodic panel and on its homogenised twin plate. The results demonstrate that the simultaneous control of the inplane, out-of-plane and their coupled behavior enables to shift a flat panel into a dome or a saddle shaped structure. Moreover, the obtained unit cells are elementary blocks to create directly 3D printable objects with shape-morphing capabilities.
\end{abstract}

Keywords: topology optimization, micro-architectured panels, shape morphing, inverse homogenization, level set method.

\section{Introduction}

Contemporary advancements of manufacturing technologies have led to the widespread adoption of materials with complex microstructures over the last decades [1-6]. These materials, constructed through sophisticated, hierarchical microstructures, overcome inherently conflicting mechanical properties in engineering practice [7,8]. Hence, it is no surprise that they are highly desirable by engineers and physical scientists.

An example of a material with periodic microstructure manufactured by classical processes is the honeycomb elastic panel $[9,10]$. It has witnessed many applications in industry due to its high strength-to-weight ratio [11] and its exceptional properties outside the elastic domain [12-14]. In the context of panels, the manufacturing of tailored micro-architecture of the material through various $3 \mathrm{D}$ printing technologies opens the way to customize the material distribution through the thickness. The wide range of novel micro-architectures will locally couple various material properties, such as extension and bending response, in what are called generally "transformation mechanisms". The underlying interest is the morphing of flat panels into three-dimensional shells, an ubiquitous mechanism found in nature with increasing technological applications [15].

In engineering, flat panels permitted the development of three-dimensional objects of complex geometries [16-18] and unleashed new functionalities for exploring harsh or inaccessible environments $[19,20]$ and delivering increasingly

\footnotetext{
${ }^{*}$ Corresponding author

Email address: andrei.constantinescu@polytechnique.edu (Andrei Constantinescu)
} 
large and complex payloads [21, 22]. However, due to their micro-structural intricacy, panel equations and associated boundary conditions are utilized and applied on a macroscopic scale where often extension-bending effects are present. Designing 3D microstructures with desired extension-bending effects is one of the aims of this work.

One of the methods designed for the analysis of micro-architectured materials is the theory of homogenization [2328]. In this theory, the effective material properties of periodic structures are defined by the analysis on a periodic cell and, in turn, these properties depend on the mechanics of constituents and the topology of the periodic structure but are independent of the external boundary conditions and applied forces. Naturally, there exists a large body of work deriving the homogenised equations of panels/plates [28-31]. For panels with thickness comparable to the length of the period, the derived effective model leads to the generalised Kirchhoff-Love thin plate model at the first order. It consists of a coupled system of equations, one equation models the in-plane behavior of the panel while the second equation models the flexural curvature. Consequently, three sets of effective coefficients are obtained that can be computed numerically once a geometry and volume fraction are determined. The first set of effective coefficients captures the in-plane panel stiffness, the second set captures the bending stiffness, and the third set captures the extension-bending effect of the panel. The extension to the next order of the asymptotic would lead to a bendinggradient model for thick plates as illustrated in [31]. Equivalent homogenization and dimension reduction that leads to the theory of Reissner-Mindlin for plates does not yet exist, to our knowledge.

When it comes to designing materials with microstructure, modern numerical methods such as shape and topology optimization [32-34] have become prevalent in this realm, leading to the design of novel complex morphologies. Nowadays, the most common techniques adopted for structural design optimization are density-based methods, e.g., the homogenization method [35, 36] and the Solid Isotropic Material with Penalization (SIMP) [33, 37], or implicit method, such as phase-field [38, 39] and the level set methods [40, 41]. For periodic materials, the overall properties can be studied using homogenization where the effective coefficients computed take into account the bulk material composition as well as the geometry layout [35]. Topology optimization using inverse homogenization exploits this fact in order to systematically identify optimal topologies and volume fractions for two-dimensional [42-46] and more recently three-dimensional periodic cell [47-51]. The works cited above, designed optimal microstructures using inverse homogenization in $2 \mathrm{D}$ or $3 \mathrm{D}$ for elastic or thermoelastic material. However, the optimal design of panels does not appear to have progressed as rapidly. One of the pioneering papers in the design of composite plates is that of [52], where the authors consider the design of extremely rigid clamped square plates. In their analysis, they consider the out-of-plane displacement of the plate without taking into account any extension-bending effects. More recently, two-scale topology optimization of composite plates was undertaken in [53]. The authors assumed that, macroscopically, the plate follows the Reissner-Mindlin theory and considered two optimization problems: inplane optimization of the periodic cell that maximizes the macroscopic stiffness of the composite plate and in-plane optimization of the periodic cell that maximizes the macroscopic displacements at prescribed nodes.

The novelty of the present work is to design thin elastic panels that simultaniously control in-plane stiffness, outof-plane bending, and extension-bending coupling. The macroscopic behavior of the panels is governed by the Kirchhoff-Love model as that is derived from the theory of homogenization in [28-31]. Building upon our previous work in $[45,46,54]$, we use inverse homogenization and a level set method coupled with the Hadamard shape derivative [40] to construct plate elastic moduli within the periodic cell. The approach is undertaken in the context of the diffuse (or smooth) interphase approach [55]. This approach entails approximating the sharp interface between material and "void" with a smooth, thin transitional layer of size $2 e$, where $e>0$ is a small number. This is primarily done for mathematical and physical reasons alike. The approach presented here allows for direct control of the extension-bending coefficient in addition to direct control of the in-plane stiffness and the out-of-plane bending stiffness.

The paper is organized as follows. In Section 2 we specify the problem setting and we present the panel's effective equations and the associate effective moduli. Section 3 is devoted to formulating the cost functional, introducing the level set method in the diffuse interface context and the discussion of the volume constraints. Section 4 presents the optimization algorithm and addresses certain algorithmic issues that arise. Section 5 deals with the implementation and discussion of several numerical examples as well as the physical meaning of the extension-bending coupling. A short summary and additional remarks in Section 6 concludes the paper. 
Notation. Throughout the paper will make use of Cartesian coordinates and of the following notation:

- Scalars are denoted by italic letters.

- Vectors, second order tensors and fourth order tensors are denoted by bold face italic letters, e.g. $\boldsymbol{u}=\left\{u_{i}\right\}_{i=1}^{3}$, $\boldsymbol{\sigma}=\left\{\sigma_{i j}\right\}_{i, j=1}^{3}$ and $\boldsymbol{C}=\left\{C_{i j k \ell}\right\}_{i, j, k, \ell=1}^{3}$.

- We adopt the Einstein summation convention, unless otherwise stated, where Latin indices range form 1 to 3 and Greek indices range from 1 to 2 .

- The average of a quantity over a region, e.g. $\mathcal{D}$, is denoted by $\langle\bullet\rangle_{\mathcal{D}}$ while by $\langle\bullet \mid \bullet\rangle$ we denote the duality product.

- The dot product between two second order tensors $\boldsymbol{A}$ and $\boldsymbol{B}$ is denoted by $\boldsymbol{A}: \boldsymbol{B}=\sum_{i, j=1}^{N} A_{i j} B_{j i}$ where $A_{i j}$ and $B_{i j}$ are the tensor components.

- The following differential operators will be used:

- $\boldsymbol{\nabla} \phi$ with components $\phi_{, i}$ denotes the gradient of the scalar field $\phi$,

- $\boldsymbol{\nabla} \boldsymbol{u}$ with components $u_{i, j}$ denotes the gradient of the vector field $\boldsymbol{u}$,

- $\boldsymbol{\nabla} \cdot \boldsymbol{u}=u_{i, i}$ denotes the divergence of the vector field $\boldsymbol{u}$,

- $\boldsymbol{\nabla} \cdot \boldsymbol{\sigma}$ with components $\sigma_{i j, j}$ denotes the divergence of the tensor field $\boldsymbol{\sigma}$.

\section{Setting of the problem}

Domain definition. The panel under consideration is occupying a bounded domain $\left.\Omega_{h}=\omega \times\right]-h / 2, h / 2\left[\subset \mathbb{R}^{3}\right.$, characterised by its neutral plane $\omega \subset \mathbb{R}^{2}$ of characteristic length $L$, and by its thickness $h$ along the $\left(O, x_{3}\right)$ axis, as sketched in Figure 1. The domain $\Omega_{h}$ is delimited by a regular boundary $\Gamma$, which is decomposed into a lateral boundary $\left.\Gamma^{l a t}=\partial \omega \times\right]-h / 2, h / 2\left[\right.$, and a top/bottom boundary $\Gamma^{ \pm}=\omega \times\{ \pm h / 2\}$.

The panel's microstructure is characterised by an in-plane periodic arrangement, composed of a large number of identical unit cells. The period, i.e., the characteristic length of a unit cell $\ell$, is assumed to be small in comparison to the characteristic size of the panel $L$. The small parameter $\epsilon=O(\ell / L) \ll O(1)$ referred to as the scale factor, expresses this difference of scales. This scale separation assumption allows one to obtain a set of homogenised plate equations as is presented in the following section. In addition, $h$ and $\ell$ are assumed to be comparable in scale, for the purposes of this work. Their ratio, denoted by $r=h / \ell=O(1)$, describes the cell's aspect ratio.

Equations at the local scale. Let $Y=[0,1]^{2} \times[-r / 2, r / 2]$ be the rescaled periodic unit cell, described by the set of coordinates $\boldsymbol{y}$ defined as follows:

$$
\boldsymbol{y}=\boldsymbol{x} / \epsilon
$$

Note that the scaling is made with respect to $\epsilon$ for both in-plane and out of the plane components. Through this choice, the rescaled unit cell's $Y$ thus preserves its aspect ratio, unlike in the monograph of Caillerie [29].

The panel is assumed to behave as a linearly elastic anisotropic body. The spatial distribution of the elastic stiffness $\boldsymbol{C}^{\epsilon}$ is expressed by:

$$
\boldsymbol{C}^{\epsilon}(\boldsymbol{x})=\frac{1}{h^{3}} \boldsymbol{C}\left(\frac{\boldsymbol{x}}{\epsilon}\right)
$$

where $\boldsymbol{C}(\boldsymbol{y})$ is an in-plane periodic, piecewise constant, isotropic fourth order tensor. The superscript $\epsilon$ is introduced to highlight the dependence on the scale factor. As proposed by Caillerie in [29], the elastic moduli are assumed to 

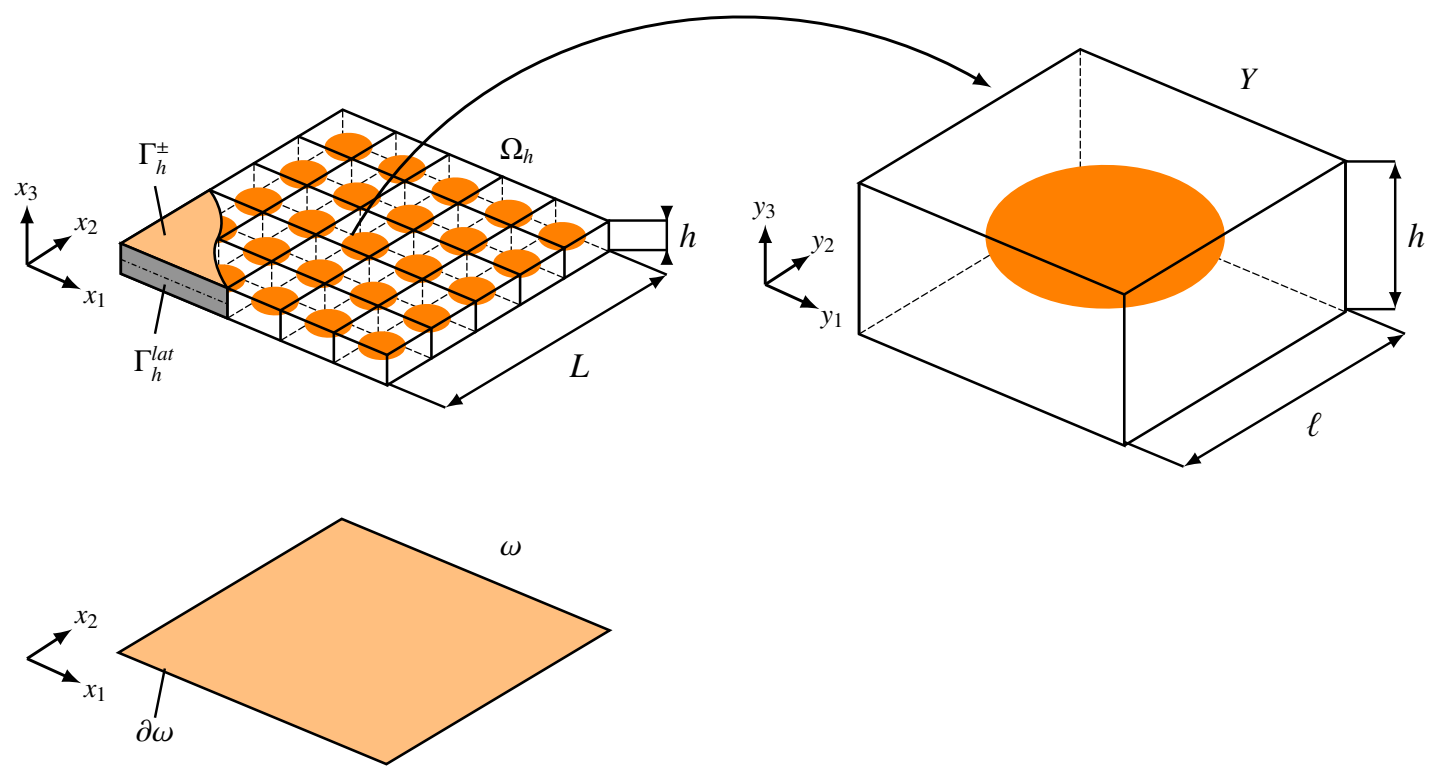

Figure 1: Architectured panel $\Omega_{h}$ with a in-plane periodic arrangement of unit cells $Y$. The cell's aspect ratio $r$ is defined as $r=h / \ell$. Concept of homogenization yields an equivalent anisotropic homogeneous plate.

depend on $h$ mainly according to $1 / h^{3}$ : as the plate gets thinner, it becomes stiffer in order to withstand the stresses that are applied to it. Moreover, the solid is submitted to surface traction $g$ at the boundary $\Gamma^{ \pm}$and a homogeneous Dirichlet boundary condition for the displacement at the boundary $\Gamma^{\text {lat }}$.

In the framework of linear elasticity, the composite panel is governed by the following set of equations and boundary conditions:

$$
\begin{cases}\boldsymbol{\nabla} \cdot \boldsymbol{\sigma}^{\epsilon}=\mathbf{0} & \text { in } \Omega_{h}, \\ \boldsymbol{\sigma}^{\epsilon}=\boldsymbol{C}^{\epsilon}(\boldsymbol{x}): \boldsymbol{\varepsilon}\left(\boldsymbol{u}^{\epsilon}\right) & \text { in } \Omega_{h}, \\ \boldsymbol{u}^{\epsilon}=\mathbf{0} & \text { on } \Gamma^{\text {lat, }} \\ \boldsymbol{\sigma}^{\epsilon} \cdot \boldsymbol{n}=\boldsymbol{g} & \text { on } \Gamma^{ \pm} .\end{cases}
$$

where $\boldsymbol{\sigma}^{\epsilon}$ is the stress field, $\boldsymbol{u}^{\epsilon}$ is the displacement field, $\boldsymbol{\varepsilon}\left(\boldsymbol{u}^{\epsilon}\right)=\frac{1}{2}\left(\boldsymbol{\nabla} \boldsymbol{u}^{\epsilon}+\nabla^{\top} \boldsymbol{u}^{\epsilon}\right)$ is the small strain tensor, and $\boldsymbol{n}$ is the external unit normal of $\Gamma^{ \pm}$.

Equations at the macroscopic scale. A classical result from homogenization theory $[28,31]$ indicate that the 3D heterogeneous problem (2.2) can be approximated at the macroscopic scale by an equivalent homogenised linearly elastic anisotropic thin plate model when the plate thickness $h$ and the characteristic cell's length $\ell$ are very small in comparison with the in-plane typical length of the plate $L$ (i.e., $l \approx t \ll L$ ). We recall that the plate problem consists in finding a plate displacement field $\boldsymbol{U}\left(x_{1}, x_{2}\right)$ and the corresponding generalised strain field $\left(\boldsymbol{\varepsilon}(\boldsymbol{U}), \chi\left(U_{3}\right)\right)$ expressed by:

$$
\varepsilon_{\alpha \beta}(\boldsymbol{U})=\frac{1}{2}\left(U_{\alpha, \beta}+U_{\beta, \alpha}\right), \quad \chi_{\alpha \beta}\left(U_{3}\right)=-U_{3, \alpha \beta},
$$

where $\epsilon_{\alpha \beta}$ is the plane strain, $\chi_{\alpha \beta}$ is the tensor of bending curvature, and a generalised stress field $(\boldsymbol{N}, \boldsymbol{M})$ on $\omega$ with $\boldsymbol{N}$ the plane stress and $\boldsymbol{M}$ the moments, satisfying the following set of equations (refer to section 8.2. in [31] for further 
details):

$$
\begin{cases}\boldsymbol{\nabla} \cdot \boldsymbol{N}+\boldsymbol{T}=\mathbf{0} & \text { in } \omega, \\ \boldsymbol{\nabla} \cdot(\boldsymbol{\nabla} \cdot \boldsymbol{M})+\boldsymbol{\nabla} \cdot \boldsymbol{Q}-T_{3}=0 & \text { in } \omega, \\ \boldsymbol{N}=\boldsymbol{A}: \boldsymbol{\varepsilon}(\boldsymbol{U})+\boldsymbol{B}: \chi\left(U_{3}\right) & \text { in } \omega, \\ \boldsymbol{M}=\boldsymbol{B}^{\top}: \boldsymbol{\varepsilon}(\boldsymbol{U})+\boldsymbol{D}: \chi\left(U_{3}\right) & \text { in } \omega, \\ \boldsymbol{U}=\mathbf{0} & \text { on } \partial \omega\end{cases}
$$

where $\boldsymbol{T}$ and $\boldsymbol{Q}$ represent the generalised external loads:

$$
\boldsymbol{T}=\int_{ \pm h / 2} \boldsymbol{g} d x_{3}, \quad \boldsymbol{Q}=\int_{ \pm h / 2} x_{3} \boldsymbol{g} d x_{3} .
$$

The elastic material behavior is expressed through the elasticity tensors $\boldsymbol{A}, \boldsymbol{B}$ and $\boldsymbol{D}$ with the following symmetries:

$$
\begin{aligned}
& A_{\alpha \beta \gamma \delta}=A_{\beta \alpha \gamma \delta}=A_{\alpha \beta \delta \gamma}=A_{\gamma \delta \alpha \beta}, \\
& B_{\alpha \beta \gamma \delta}=B_{\beta \alpha \gamma \delta}=B_{\alpha \beta \delta \gamma}, \\
& D_{\alpha \beta \gamma \delta}=D_{\beta \alpha \gamma \delta}=D_{\alpha \beta \delta \gamma}=D_{\gamma \delta \alpha \beta},
\end{aligned}
$$

which guarantee symmetry of strains and stresses as well as the existence of an energy potential. In more precise terms, $\boldsymbol{A}$ describes the in-plane behavior, $\boldsymbol{D}$ describes the bending behavior, and their coupling is expressed through $\boldsymbol{B}$. Note that in most engineering applications, where panels feature symmetric geometry and material distribution along the thickness, normal and shear behavior get uncoupled for the membrane part, yielding $\boldsymbol{B}=\mathbf{0}$. The complementary behavior is investigated here, i.e., we aim at designing panels with exceptional extension-bending coupling effect.

Effective plate moduli. As first developed by Caillerie in [29], the effective tensors $\boldsymbol{A}^{*}, \boldsymbol{B}^{*}$ and $\boldsymbol{D}^{*}$ can be derived through periodic homogenization theory (see also [28, 31]). This procedure is schematically depicted in Figure 1. Moreover, the effective thin plate elasticity tensors described above can be computed in their energy form from the solutions of elasticity problems with prescribed mean strain modes. More precisely, the effective coefficients $\boldsymbol{A}^{*}, \boldsymbol{B}^{*}$ and $\boldsymbol{D}^{*}$ are expressed, component-wise, as:

$$
\begin{aligned}
& A_{\alpha \beta \gamma \delta}^{*}=\frac{r}{|Y|} \int_{Y}\left(\boldsymbol{E}^{\alpha \beta}+\boldsymbol{\varepsilon}_{\boldsymbol{y}}\left(\boldsymbol{w}^{\alpha \beta}\right)\right): \boldsymbol{C}(\boldsymbol{y}):\left(\boldsymbol{E}^{\gamma \delta}+\boldsymbol{\varepsilon}_{\boldsymbol{y}}\left(\boldsymbol{w}^{\gamma \delta}\right)\right) d \boldsymbol{y}, \\
& B_{\alpha \beta \gamma \delta}^{*}=\frac{r}{|Y|} \int_{Y}\left(\boldsymbol{X}^{\alpha \beta}+\boldsymbol{\varepsilon}_{\boldsymbol{y}}\left(\boldsymbol{p}^{\alpha \beta}\right)\right): \boldsymbol{C}(\boldsymbol{y}):\left(\boldsymbol{E}^{\gamma \delta}+\boldsymbol{\varepsilon}_{\boldsymbol{y}}\left(\boldsymbol{w}^{\gamma \delta}\right)\right) d \boldsymbol{y}, \\
& D_{\alpha \beta \gamma \delta}^{*}=\frac{r}{|Y|} \int_{Y}\left(\boldsymbol{X}^{\alpha \beta}+\boldsymbol{\varepsilon}_{\boldsymbol{y}}\left(\boldsymbol{p}^{\alpha \beta}\right)\right): \boldsymbol{C}(\boldsymbol{y}):\left(\boldsymbol{X}^{\gamma \delta}+\boldsymbol{\varepsilon}_{\boldsymbol{y}}\left(\boldsymbol{p}^{\gamma \delta}\right)\right) d \boldsymbol{y} .
\end{aligned}
$$

In the above equations, $\boldsymbol{E}^{\alpha \beta}$ (resp. $\boldsymbol{X}^{\alpha \beta}$ ) are the prescribed mean in-plane (resp. flexural) strain modes on the unit cell, depicted in Figure 2. They are chosen to form a vector basis in the space of second order symmetric tensors and are expressed as:

$$
\boldsymbol{E}^{\alpha \beta}=\frac{1}{2}\left(\delta_{i \alpha} \delta_{j \beta}+\delta_{i \beta} \delta_{j \alpha}\right) \boldsymbol{e}_{i} \otimes \boldsymbol{e}_{j}, \quad \boldsymbol{X}^{\alpha \beta}=\frac{y_{3}}{2}\left(\delta_{i \alpha} \delta_{j \beta}+\delta_{i \beta} \delta_{j \alpha}\right) \boldsymbol{e}_{i} \otimes \boldsymbol{e}_{j}
$$

$\boldsymbol{w}^{\alpha \beta}$ and $\boldsymbol{p}^{\alpha \beta}$ are displacement fields, solutions of the 6 local problems (see Figure 2). Let us further remark, that the tensor $\boldsymbol{E}^{\alpha \beta}$ is constant and the tensor $\boldsymbol{X}^{\alpha \beta}$ depends on the vertical position which permits to define the local periodic fields. By introducing the functional space $\mathcal{V}(Y):=\left\{v \in H^{1}(Y) \mid v\right.$ is $\left(y_{1}, y_{2}\right)$-periodic, $\left.\langle v\rangle_{Y}=0\right\}$, the cell problems can 


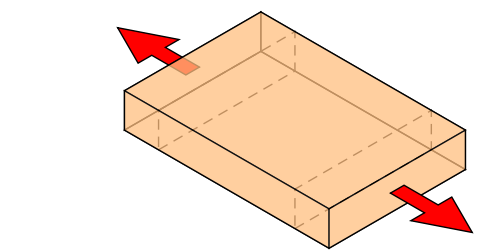

$\leftarrow_{e_{1}}^{e_{3}}$

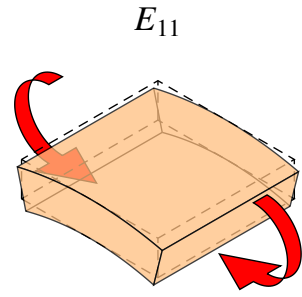

$X_{11}$

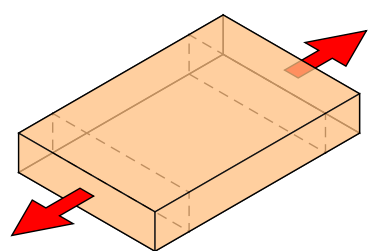

$E_{22}$

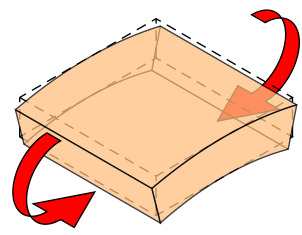

$X_{22}$

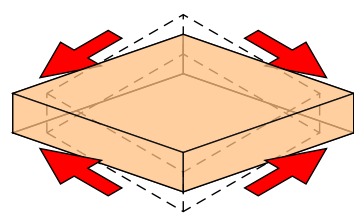

$E_{12}$

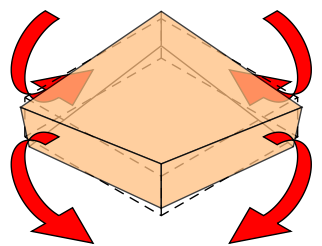

$X_{12}$

Figure 2: Strain modes imposed to solve the local problems (2.7) and (2.8). The undeformed unit cell is represented by dotted lines, whereas the deformed configuration is indicated by solid lines. The first row displays the three in-plane modes, namely two tensions and one in-plane shear, whereas the second row corresponds to the out of the plane modes, namely two bending and one shear bending.

be expressed in their variational formulation:

Find $\boldsymbol{w}^{\gamma \delta} \in \mathcal{V}(Y)$ such that:

$$
\int_{Y}\left(\boldsymbol{E}^{\gamma \delta}+\boldsymbol{\varepsilon}_{\boldsymbol{y}}\left(\boldsymbol{w}^{\gamma \delta}\right)\right): \boldsymbol{C}(\boldsymbol{y}): \boldsymbol{\varepsilon}_{\boldsymbol{y}}(\boldsymbol{\varphi}) d \boldsymbol{y}=0 \quad \forall \boldsymbol{\varphi} \in \mathcal{V}(Y)
$$

Find $\boldsymbol{p}^{\gamma \delta} \in \mathcal{V}(Y)$ such that:

$$
\int_{Y}\left(X^{\alpha \beta}+\boldsymbol{\varepsilon}_{y}\left(\boldsymbol{p}^{\alpha \beta}\right)\right): \boldsymbol{C}(\boldsymbol{y}): \boldsymbol{\varepsilon}_{\boldsymbol{y}}(\boldsymbol{\psi}) d \boldsymbol{y}=0 \quad \forall \boldsymbol{\psi} \in \mathcal{V}(Y)
$$

We point out that the first local problem (2.7) is concerned with the in-plane deformation modes, while the local problem (2.8) corresponds to the out-of-plane bending modes.

\section{Optimization problem}

Cost functional. The design domain of the optimization is the periodic cell $Y \in[0,1]^{2} \times[-r / 2, r / 2]$ defined in the previous section. For expediency, we consider only a two-phase material with the extension to multi-phase material being handled as in e.g. [55], [45]. The cell may be decomposed into a strong phase $S$ (typically the material phase), that will also be referred to as shape, and weak phase $\bar{S}$ (which represents the void), separated by a interphase $\Gamma^{S}$. Moreover, we assume that $(S, \bar{S}) \subset Y$ are smooth, open, bounded subsets and define the set of admissible shapes,

$$
\mathcal{U}_{a d}:=\left\{S \subset Y \text { is open, bounded, and smooth }\left|\rho_{m} \leq\right| S \mid \leq \rho_{M}\right\},
$$

where $|S|$ corresponds to the shape's volume fraction, while $\rho_{m}$ and $\rho_{M}$ are two real numbers ranging between 0 and 1. We are ready to define an objective function $\mathcal{J}$, to be minimised over all possible admissible shapes, as a sum of weighted Euclidean norms:

$$
\mathcal{J}(S)=\frac{1}{2} \eta_{\alpha \beta \gamma \delta}^{A}\left(A_{\alpha \beta \gamma \delta}^{*}-A_{\alpha \beta \gamma \delta}^{\mathrm{target}}\right)^{2}+\frac{1}{2} \eta_{\alpha \beta \gamma \delta}^{B}\left(B_{\alpha \beta \gamma \delta}^{*}-B_{\alpha \beta \gamma \delta}^{\mathrm{target}}\right)^{2}+\frac{1}{2} \eta_{\alpha \beta \gamma \delta}^{D}\left(D_{\alpha \beta \gamma \delta}^{*}-D_{\alpha \beta \gamma \delta}^{\mathrm{target}}\right)^{2},
$$

where $\boldsymbol{A}^{\text {target }}, \boldsymbol{B}^{\text {target }}$ and $\boldsymbol{D}^{\text {target }}$ denote given target thin plate tensor values, while $\boldsymbol{\eta}^{A}, \boldsymbol{\eta}^{B}$ and $\boldsymbol{\eta}^{D}$ are the weight coefficients carrying the same type of symmetry as their respective tensor. Consequently, the topology optimization 
problem under consideration reads:

$$
\begin{aligned}
& \inf _{S \subset \mathcal{U}_{a d}} \mathcal{J}(S), \\
& \text { subject to (2.7) and (2.8). }
\end{aligned}
$$

We have chosen an interval for the volume fraction in $\mathcal{U}_{a d}$ since it is not a priori possible to guess a compatible target volume fraction for a target stiffness tensor. If the prescribed material volume fraction is set too low, the target stiffness could fall outside the range of achievable tensors [56, 57], resulting in a final shape with undesired effects (see for example the gap between the target and the obtained results in the two first final shapes of [46]). This is an even bigger issue considering that to our knowledge, variational bounds for elastic thin plates have not yet been studied. Conversely, in the case where prescribed material volume fraction is relatively high, the algorithm may converge to shapes that are excessively bulky (e.g. large blocks connected with thin hinges) or in the worst case scenario, the algorithm would leave some unconnected material phases (islands) in the final microstructure.

Note that the interval volume constraint is enforced using an augmented Lagrangian method, which is detailed in Appendix B

\subsection{Coupling shape sensitivity with a level set description}

Shape sensitivity analysis. Shape optimization problems are often not compatible with discrete or zero-order methods [58], rather, they are addressed using gradient-based continuous optimization algorithms. The notion of gradient for shape optimization problems, namely the method for describing variations of a shape, is based on Hadamard's boundary variation method which has become standard in the literature [59], [60, section 2.6], [34, Chapter 6], [61, Chapter 4].

Henceforth, the characterization of different phases is described using a level set function and as a consequence, a descent direction can be obtained by computing the shape derivative of $\mathcal{J}(S)$ within the classical shape sensitivity framework of Hadamard. A short description of the level set is provided next, while the detailed derivation of the shape derivative of $\mathcal{J}(S)$ can be found in Appendix A for the readers convenience.

Shape representation by the level set method. Developed by Osher and Sethian [62], the level set method is a technique for tracking interfaces which are implicitly defined via the zero level set of an auxiliary scalar function $\phi$. The key idea consists in replacing the usual representation of a domain $\omega \subset Y$ by an implicit representation, as the negative sub-domain of an auxiliary scalar function $\phi$ defined on the whole space $Y$, as illustrated in Figure 3. More precisely, the shape $\omega$ is known via a function $\phi: Y \rightarrow \mathbb{R}$ defined in Equation (3.4).

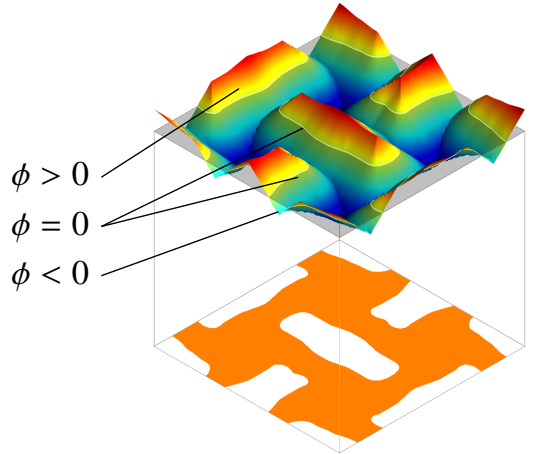

$$
\left\{\begin{array}{lllr}
\phi(\boldsymbol{y})<0 & \text { if } & \boldsymbol{y} \in S & \text { (material) } \\
\phi(\boldsymbol{y})=0 & \text { if } & \boldsymbol{y} \in \Gamma^{S} & \text { (boundary) } \\
\phi(\boldsymbol{y})>0 & \text { if } & \boldsymbol{y} \in \bar{S}=Y \backslash S & \text { (void) }
\end{array}\right.
$$

Figure 3: Representation of the shape in a two-dimensional unit cell: a 3D representation of the level set sliced by the plane $\phi=0$ projection of the level set on the Cartesian plane (center), characteristic sets defined by the level set, i.e. void and material phases and their reciprocal boundary. 
A pseudo time $t \in \mathbb{R}^{+}$is defined to characterise the evolution of the shape $S(t)$ via its corresponding level set $\phi(y(t), t)$. Initially the interphase of the shape is described by $\phi(y)=0$ if $y \in \Gamma^{S}$. Consequently, for any pseudo time $t, \boldsymbol{y}(t) \in \Gamma^{S}(t)$ satisfies $\phi(y(t), t)=0$. Differentiating with respect to the pseudo time $t$ yields:

$$
\frac{d \phi}{d t}(\boldsymbol{y}(t), t)=0 \quad \Rightarrow \quad \frac{\partial \phi}{\partial t}(\boldsymbol{y}, t)+\boldsymbol{\theta}(\boldsymbol{y}, t) \cdot \boldsymbol{\nabla} \phi(\boldsymbol{y}, t)=0
$$

where $\boldsymbol{\theta}=\frac{\partial y}{d t}$ is the velocity field of the interphase $\Gamma^{S}(t)$. For a small variation of the shape, its evolution is completely described by the normal component of velocity field $\boldsymbol{\theta}$, as justified by the Hadamard structure theorem [34, 59]. Hence, after introducing the normal component of $\boldsymbol{\theta}: V=\boldsymbol{\theta} \cdot \boldsymbol{n}$, eq. (3.5) can be written as:

$$
\frac{\partial \phi}{\partial t}(\boldsymbol{y}, t)+V(\boldsymbol{y}, t)|\boldsymbol{\nabla} \phi(\boldsymbol{y}, t)|=0 \quad \forall t, \forall \boldsymbol{y} \in Y,
$$

which takes form of the Hamilton-Jacobi equation.

Smooth interphase approach. In each phase, the material properties are characterised by an isotropic elastic tensor $\boldsymbol{C}^{n}$ ( $n=S$ refers to the stronger phase, $n=\bar{S}$ refer to the weaker one). Assuming a sharp interface would induce a discontinuity of $\boldsymbol{C}^{n}$. For physical and mathematical reasons it is often desirable to model the interphase as a smooth, transitional layer of thickness $2 e$, where $e$ is a small positive parameter. Following the ideas in [55], the level set function serves as a base to define the smooth local stiffness tensor $\boldsymbol{C}^{e}$ in $Y$ as a regular interpolation between the strong phase and the weak phase. The transition from a sharp to a smooth interface is achieved first by redistancing the level set $\phi$ to become the signed distance function $d_{S}$ to the interface boundary $\Gamma^{S}$. Then, using a smooth Heaviside type of function we describe the distribution of elastic properties in a smooth way. The Heaviside function $\mathcal{H}_{e}$ used in this study reads:

$$
\mathcal{H}_{e}(t)= \begin{cases}0 & \text { if } t<-e \\ \frac{1}{2}\left(1+\frac{t}{e}+\frac{1}{\pi} \sin \left(\frac{\pi t}{e}\right)\right) & \text { if }|t| \leq e \\ 1 & \text { if } t>e\end{cases}
$$

The choice of the regularizing function $\mathcal{H}_{e}$ is not unique: it is possible to use other type of regularizing functions (see [42] for instance). Hence, the properties of the material occupying the unit cell Y are then defined as a smooth interpolation between the tensors $\boldsymbol{C}^{S}$ and $\boldsymbol{C}^{\bar{S}}$ :

$$
\boldsymbol{C}^{e}=\mathcal{H}_{e}\left(d_{S}\right)\left(\boldsymbol{C}^{\bar{S}}-\boldsymbol{C}^{S}\right)+\boldsymbol{C}^{S}
$$

and the material volume fraction $|S|$ is defined by:

$$
|S|=\frac{1}{|Y|} \int_{Y}\left(1-\mathcal{H}_{e}\right) d \boldsymbol{y} .
$$

Lastly, from the computations in Appendix A, the expressions for the shape derivative of $\mathcal{J}(S)$ in direction of the velocity field $\boldsymbol{\theta}$ under the approximation of thin smooth interphase reads:

$$
\mathcal{J}^{\prime}(S)(\boldsymbol{\theta})=-\int_{\Gamma^{S}}\left(f_{A}(\boldsymbol{s})+f_{B}(\boldsymbol{s})+f_{D}(\boldsymbol{s})\right) \boldsymbol{\theta} \cdot \boldsymbol{n} d \boldsymbol{s}
$$

where:

$$
\begin{aligned}
& f_{A}(\boldsymbol{s})=\frac{r}{|Y|} \eta_{\alpha \beta \gamma \delta}^{A}\left(A_{\alpha \beta \gamma \delta}^{*}\left(d_{S}\right)-A_{\alpha \beta \gamma \delta}^{\mathrm{target}}\right)\left(\boldsymbol{E}^{\gamma \delta}+\boldsymbol{\varepsilon}_{\boldsymbol{y}}\left(\boldsymbol{w}^{\gamma \delta}\right)\right):\left(\boldsymbol{C}^{\bar{S}}-\boldsymbol{C}^{S}\right):\left(\boldsymbol{E}^{\alpha \beta}+\boldsymbol{\varepsilon}_{\boldsymbol{y}}\left(\boldsymbol{w}^{\alpha \beta}\right)\right), \\
& f_{B}(\boldsymbol{s})=\frac{r}{|Y|} \eta_{\alpha \beta \gamma \delta}^{B}\left(B_{\alpha \beta \gamma \delta}^{*}\left(d_{S}\right)-B_{\alpha \beta \gamma \delta}^{\mathrm{target}}\right)\left(\boldsymbol{E}^{\gamma \delta}+\boldsymbol{\varepsilon}_{\boldsymbol{y}}\left(\boldsymbol{w}^{\gamma \delta}\right)\right):\left(\boldsymbol{C}^{\bar{S}}-\boldsymbol{C}^{S}\right):\left(\boldsymbol{X}^{\alpha \beta}+\boldsymbol{\varepsilon}_{\boldsymbol{y}}\left(\boldsymbol{p}^{\alpha \beta}\right)\right), \\
& f_{D}(\boldsymbol{s})=\frac{r}{|Y|} \eta_{\alpha \beta \gamma \delta}^{D}\left(D_{\alpha \beta \gamma \delta}^{*}\left(d_{S}\right)-D_{\alpha \beta \gamma \delta}^{\mathrm{target}}\right)\left(\boldsymbol{X}^{\gamma \delta}+\boldsymbol{\varepsilon}_{\boldsymbol{y}}\left(\boldsymbol{p}^{\gamma \delta}\right)\right):\left(\boldsymbol{C}^{\bar{S}}-\boldsymbol{C}^{S}\right):\left(\boldsymbol{X}^{\alpha \beta}+\boldsymbol{\varepsilon}_{\boldsymbol{y}}\left(\boldsymbol{p}^{\alpha \beta}\right)\right) .
\end{aligned}
$$


Hence, a descent direction can always be selected by choosing $\boldsymbol{\theta}=\left(f_{A}(\boldsymbol{s})+f_{B}(\boldsymbol{s})+f_{D}(\boldsymbol{s})\right) \boldsymbol{n}$.

As a final comment, let us remark that the smooth interface approach affects any numerical integration and its associated discretization scheme used in all problems.

\subsection{Volume constraint}

The result in eq. (3.10) corresponds to the unconstrained problem. To ensure that $S \subset \mathcal{U}_{\text {ad }}$, we rely on an augmented Lagrangian approach to enforce a two-sided inequality constraints [63], [64, Chapter 17]. Hence, the optimization problem (3.3) is a constraint-free minimization of a (Lagrangian-like) weighted sum of the cost functional $\mathcal{J}(S)$ and the constraint $\mathcal{P}(S, \lambda, \mu)$ that reads:

$$
\inf (\mathcal{J}(S)+\mathcal{P}(S, \lambda, \mu))
$$

where the constraint is expressed as follows:

$$
\mathcal{P}(S, \lambda, \mu)=\min _{\rho_{m} \leq|S|-v \leq \rho_{M}}\left(\lambda v+\frac{\mu}{2}|v|^{2}\right) .
$$

In the above equation, $v$ is a slack variable, $\lambda$ is the Lagrange multiplier for the volume constraint and $\mu$ is a penalty paramater. A brief presentation on used schemes to update these parameters during the optimization process is provided in Appendix B. As indicate in eq. (3.11), the constraint gives rise to an additional term in the shape derivative of $\mathcal{J}(S)$. Let us denote by $\mathcal{P}^{\prime}(S, \lambda, \mu)$ the shape derivative of the volume constraint $\mathcal{P}(S, \lambda, \mu)$ in the direction $\boldsymbol{\theta}$. Under the approximation of thin smooth interphase, $\mathcal{P}^{\prime}(S, \lambda, \mu)$ is expressed as follows (see Appendix B):

$$
\mathcal{P}^{\prime}(S, \lambda, \mu)(\boldsymbol{\theta})=-\int_{\Gamma^{S}} f_{P}(\boldsymbol{s}) \boldsymbol{\theta} \cdot \boldsymbol{n} d \boldsymbol{s},
$$

where

$$
f_{P}(\boldsymbol{s})= \begin{cases}\lambda^{k}+\left(|S|-\rho_{M}\right) \mu^{k} & \text { if } \lambda^{k}+\mu^{k}\left(|S|-\rho_{M}\right)>0 \\ \lambda^{k}+\left(|S|-\rho_{m}\right) \mu^{k} & \text { if } \lambda^{k}+\mu^{k}\left(|S|-\rho_{m}\right)<0 \\ 0 & \text { otherwise. }\end{cases}
$$

Note the above expression has the same " $\boldsymbol{- \theta} \cdot \boldsymbol{n}$ " form of eq. (3.10), which means that a descent direction can be found in similar manners.

\subsection{Extension and regularization of the velocity field and descent direction}

Although eq. (3.6) for the advection of the level set function is solved in the whole domain $Y$, shape sensitivity analysis provides us with a shape gradient defined only on the boundary of the domain $\Gamma^{S}$. Since the boundary is not explicitly discretized in our case, we can assume that the normal velocity $V$ is defined for the nodes of the elements that are crossed by the zero level set. Then, one possibility is to consider $V=0, \forall y \in Y \backslash \Gamma^{S}$. Unfortunately, this choice would limit the movement of the boundary to small distance, which would result in an increased number of iterations until convergence, and thus a slower algorithm. A remedy to this inconvenience is to extend the velocity field in all the domain. At the same time, it would be numerically beneficial to smooth a bit the shape gradient, but in a way that guarantees the descent nature of the new advection velocity. The sequel describes one way to combine these two requirements. Initially, the shape derivative has the form:

$$
\mathcal{J}^{\prime}(S)(\boldsymbol{\theta})=\int_{\Gamma^{S}}-\boldsymbol{\theta} \cdot \boldsymbol{n}\left(\sum f(\boldsymbol{s})\right) d \boldsymbol{s}
$$


or, for an advection velocity of the type $\theta(s)=V(s) n(s)$,

$$
\mathcal{J}^{\prime}(S)(V \boldsymbol{n})=\int_{\Gamma^{S}}-V(\boldsymbol{s})\left(\sum f(\boldsymbol{s})\right) d \boldsymbol{s}
$$

Instead of choosing $V(s)=-f(s)$, we can solve the variational formulation for $V \in H^{1}(Y)$ :

$$
\int_{Y}\left(\alpha^{2} \boldsymbol{\nabla} V \cdot \boldsymbol{\nabla} W+W V\right) d \boldsymbol{y}=\mathcal{J}^{\prime}(S)(W \boldsymbol{n}) \quad \forall W \in H^{1}(Y)
$$

where $\alpha>0$ is a positive scalar (of the order of the mesh size) to control the regularization width and take $W=-V$. This operation reveals that:

$$
\mathcal{J}^{\prime}(S)(V \boldsymbol{n})=-\int_{Y}\left(\alpha^{2}|\nabla V|^{2}+V^{2}\right) d \boldsymbol{y}
$$

which guarantees again a descent direction for $\mathcal{J}$.

\section{Optimization algorithm}

The numerical algorithm used is adapted from [40] accounting for the additional local problem that is needed to compute the effective coefficients of the composite panel.

Data: Initialize a level set function $\phi_{0}$ corresponding to an initial shape $S^{0}$;

for $k \geq 0$ iterate until convergence do

Redistance $\phi_{k}$ into the signed distance function $d_{S^{k}}$ for stability reasons;

Compute the local solutions $\boldsymbol{w}^{m \ell}, \boldsymbol{p}^{m \ell}$ for $m, \ell=1,2$ by solving (2.7), (2.8) for the domain $S^{k}$;

Update the Lagrange multiplier $\lambda^{k}$ and penalty parameters $\mu^{k}$;

Compute the shape gradient $\mathcal{J}\left(S^{k}\right)\left(\boldsymbol{\theta}^{k}\right)$ for the domain $S^{k}$ using eq. (3.10);

Deform the domain $S^{k}$ by solving the Hamilton-Jacobi equation (3.6);

// Shape $S^{k+1}$ is characterized by the level set $\phi_{k+1}$ after a time step $\Delta t_{k}$

// The time step $\Delta t_{k}$ is chosen so that $\mathcal{J}\left(S^{k+1}\right) \leq \mathcal{J}\left(S^{k}\right)$

end

Algorithm 1: Major steps of the algorithm in [40] adapted to thin composite panels.

Stopping criterion. The optimization is assumed to be terminated when 200 iterative steps are reached, or else, when the time step $\Delta t_{k}$ in the Hamilton-Jacobi equations becomes too small: $\Delta t_{k}<t_{\text {lim }}=10^{-8}$. In this case, the code reached a local minimum and cannot find a descent direction.

Algorithmic issues. As we already mentioned in the previous section, it is well known that problems of designing optimal microstructures do not possess a global minimum [34]. As a result, initial starting shapes/guesses have a considerable effect on the final design of the microstructure. Furthermore, they may lead the algorithm towards nonphysical shapes (e.g., disconnected materials island in a void matrix), or to shapes representing local minima, yet very far from the target. In this case, we can always restart the algorithm, either using different initial guesses or using a different set of algorithmic parameters (like a different time step $\Delta t_{k}$, different penalization parameter $\mu_{k}$ ).

Let us further note, that even though the notion of shape derivative does not permit nucleations, the level set method can naturally combine two adjacent "holes" into one by breaking the "barrier" between them, thus effectively changing the topology of the structure. This is why in practice, it is desirable to start with an initial guess that contains a large 


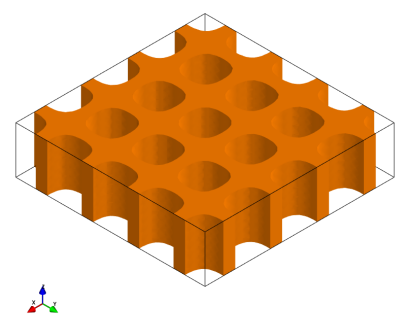

(a)

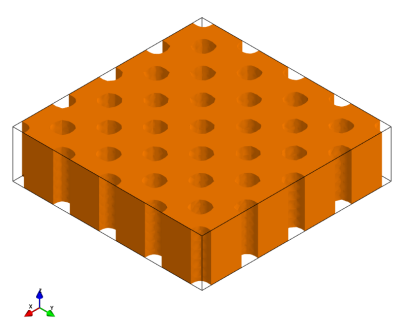

(b)

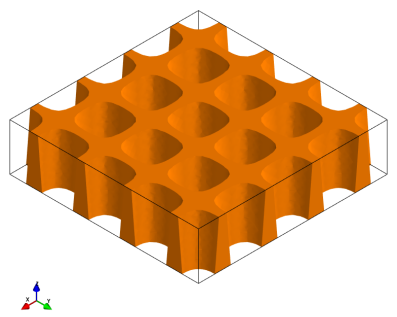

(c)

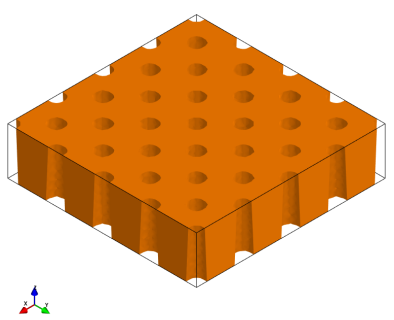

(d)

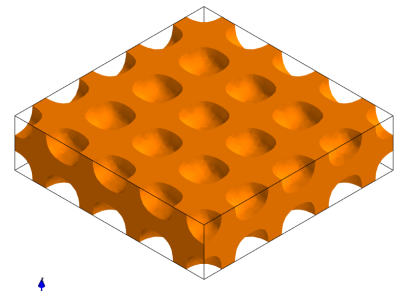

(e)

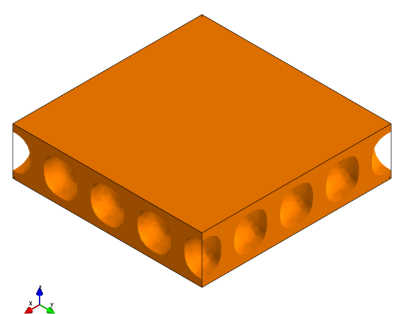

(f)

Figure 4: Initial shapes. (a) Square pattern of cylindrical micro-perforations. (b) Diagonal pattern of cylindrical micro-perforations. (c) Square pattern of conic micro-perforations. (d) Diagonal pattern of conic micro-perforations. (e) Straight bubble pattern. (f) Another straight bubble pattern, but shifted.

number of nucleations [40, 41]. However, as explained in [65], the topological change may induce an increase of the objective function $\mathcal{J}$, which we allow (up to a small tolerance) in the hope that afterwards the algorithm will locate a better shape. In practice, since most changes of topology occur in the first few iterations, the tolerance for accepting increases of the objective function $\mathcal{J}$ is defined at the $k^{\text {th }}$ iteration as:

$$
\mathcal{J}\left(S^{k+1}\right)<\mathcal{J}\left(S^{k}\right)\left(1+\eta_{\text {tol }} \exp (-0.1 k)\right)
$$

where $\eta_{\text {tol }}$ is a parameter set to a small value ( $\eta_{\text {tol }}=0.05$ in our scripts). The decreasing exponential reduces the tolerance over the iterations: until iteration 16, an increase by $1 . e-2$ is accepted, while after 40 iterations, an increase by $1 . e-3$ is no longer accepted.

Lastly, in order to discuss the influence of the initial shapes on the final designs, six kinds of initial designs displayed in Figure 4 were tested. Initial designs can be straight or diagonal patterns with various micro-perforations. The initial design Figure 4(a) and (b) feature cylinder inclusions, Figure 4(c) and (d) feature cone inclusions, Figure 4(e) and (f) feature circular inclusions. The number and the size of micro-perforations can be varied to tune the initial volume fraction.

\section{Numerical results}

In the following examples, the elementary cell $Y$ is a rectangular box of dimensions $1 \times 1 \times 0.25$ (hence the aspect ratio of the length scale $r=1 / 4)$. It is meshed with a structured symmetric grid of $100 \times 100 \times 25$ quadrangular, each formed of four equa linear tetrahedron elements for the example 1 ; a structured symmetric grid of $50 \times 50 \times 12$ linear tetrahedron elements for the examples 2 to 4 . The choice to test different mesh size enables to appreciate the effects on the computational time required and its capacity to generate new morphological structures with prescribed behavior. We recall that the distribution of elastic properties are defined by eq. (3.8). The material properties in each 
phases, $S$ and $\bar{S}$ are characterised by an isotropic fourth order tensor:

$$
\boldsymbol{C}^{n}=\frac{E^{n}}{1+v^{n}} \boldsymbol{I}_{4}+\frac{E^{n} v^{n}}{\left(1+v^{n}\right)\left(1-2 v^{n}\right)} \boldsymbol{I}_{2} \otimes \boldsymbol{I}_{2} \quad n \in\{S, \bar{S}\}
$$

where $\boldsymbol{I}_{2}$ is a second order identity matrix, and $\boldsymbol{I}_{4}$ is the identity fourth order tensor acting on symmetric matrices. The material properties are normalised as follows: the Young's modulus $E$ was set to $E^{S}=0.91 \mathrm{MPa}$ for the strong phase (material) and $E^{\bar{S}}=0.91 \times 10^{-4} \mathrm{MPa}$ for the weak phase (ersatz). The Poisson's ratio was set to $v=0.3$ for both phases. A homogeneous plate made of material $\boldsymbol{C}^{S}$ (resp. $\boldsymbol{C}^{\bar{S}}$ ) features an effective in-plane behavior $A_{1111}^{*}=A_{2222}^{*}=r$ (resp. $A_{1111}^{*}=A_{2222}^{*}=10^{-4} r$ ).

All computations were carried out using an in house programming. The elasticity problems (2.7) and (2.8) are solved using the finite element solver Cast3M. The Hamilton-Jacobi equation (3.6) is solved using the method of characteristics using the advect package developed in [66]. The re-distancing of the level set is undertaken using the mshdist package developed in [67].

Setting the target stiffness. The simultaneous in-plane, out-of-plane and their coupled behavior permits to program an out-of-plane response that results in either a dome shaped structure or a saddle shaped structure under the action of in-plane loading. As the primary interest in this work is the stretching-bending response of the panels, all shear coefficients, namely $A_{1212}^{*}, B_{1212}^{*}$ and $D_{1212}^{*}$ were left free and are denoted by a star, the controlled coefficients are therefore:

$$
C^{\mathrm{target}}=\left[\begin{array}{ccc|ccc}
A_{1111}^{*} & A_{1122}^{*} & \star & B_{1111}^{*} & B_{1122}^{*} & \star \\
A_{1122}^{*} & A_{2222}^{*} & \star & B_{2211}^{*} & B_{2222}^{*} & \star \\
\star & \star & \star & \star & \star & \star \\
\hline B_{1111}^{*} & B_{2211}^{*} & \star & D_{1111}^{*} & D_{1122}^{*} & \star \\
B_{1122}^{*} & B_{2222}^{*} & \star & D_{1122}^{*} & D_{2222}^{*} & \star \\
\star & \star & \star & \star & \star & \star
\end{array}\right]
$$

Moreover, by methodically tuning the weights of the cost functional (3.2) permits to prioritize certain crucial components at the expense of others. Furthermore, all numerical examples reported in the sequel target an elastic tensor exhibiting "quadratic symmetry", i.e. $A_{1111}^{*}=A_{2222}^{*}$ and $D_{1111}^{*}=D_{2222}^{*}$. This simplification, albeit fundamental, demonstrates the capability of the code to discriminate local solutions with general orthotropic behavior. Additionally, we point out that the values of the coefficients in $\boldsymbol{A}^{*}$ are usually much larger than the ones in $\boldsymbol{B}^{*}$ and $\boldsymbol{D}^{*}$. The difference in scale must be corrected through the weights $\eta_{A}, \eta_{B}$ and $\eta_{D}$, otherwise if $O\left(\eta_{A}\right)=O\left(\eta_{B}\right)=O\left(\eta_{D}\right)$, the cost functional $\mathcal{J}(S)$ in (3.2) can be approximated during the first iterations:

$$
\mathcal{J}(S) \approx \frac{1}{2}\left\|\boldsymbol{A}^{*}-\boldsymbol{A}^{\text {target }}\right\|_{\eta_{A}}^{2}
$$

and, therefore, the algorithm essentially satisfies the prescribed in plane behavior $\boldsymbol{A}^{\text {target }}$ neglecting $\boldsymbol{B}^{\text {target }}$ and $\boldsymbol{D}^{\text {target }}$. Our experiences concluded that an optimal choice for the weights is: $10^{2} O\left(\eta_{A}\right)=O\left(\eta_{B}\right)=O\left(\eta_{D}\right)$.

Comparison between the full model and the homogeneous equivalent. To demonstrate the effect of the extensionbending coupling, we simulate the deformation of an architectured panel loaded in tension. Periodic assemblages consisting in $4 \times 5$ (resp. $5 \times 4$ ) unit cells were considered for the tensile specimen along direction $\boldsymbol{e}_{1}$ (resp. along direction $\boldsymbol{e}_{2}$ ). A sketch of the setting, provided in Figure 5(a), describes the boundary conditions enforced on the lateral borders of the panel. The calculations are conducted with symmetry boundary conditions on the bottom and left side of the plate. The specimen is loaded by an imposed displacement on the mid-line of its right side (the red line Figure 5(a)). Furthermore, all vertices belonging to the right face with the same in-plane coordinate are assumed to move as a rigid body (this is illustrated by the blue lines in Figure 5(a)). As observed in our previous work [54], hard-clamp boundary conditions cancel out the bending response of the specimen in tension. It is thanks to this 


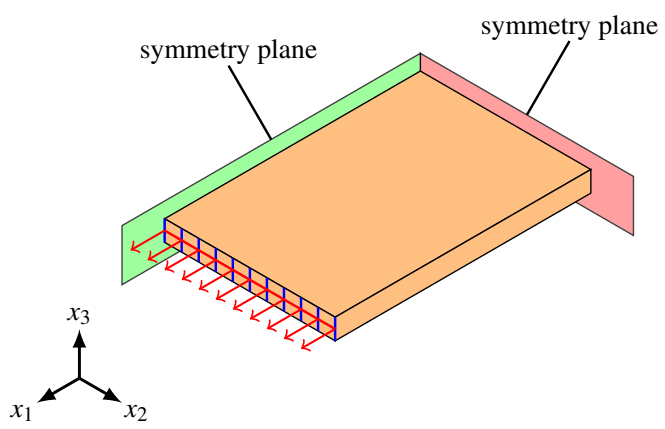

(a)

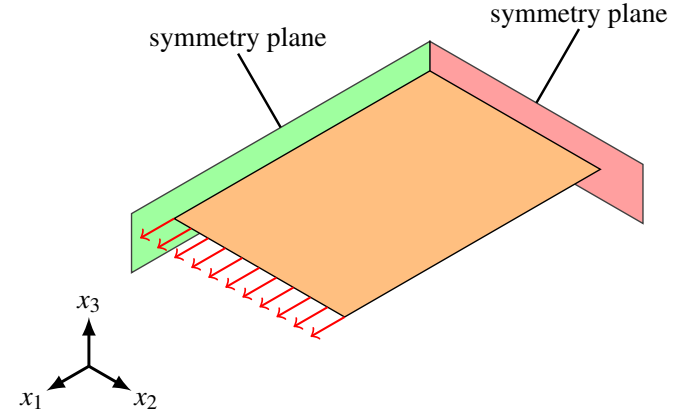

(b)

Figure 5: Numerical problem with given boundary conditions for a tensile loading along the direction $\boldsymbol{e}_{1}$. The case for tensile loading along the direction $\boldsymbol{e}_{2}$ is analoguous. The simulations account for the plane symmetries in the specimen and are performed on a quarter of the geometry. (a) Analysis performed on the complete structure. The displacement component $u_{1}$ is imposed on the mid plane represented by the red line. The blue lines indicate a rigid body kinematics applied along the thickness. (b) Analysis performed on the equivalent homogeneous thin plate, meshed with a structured pattern composed of $50 \times 40$ linear triangle elements. The amplitude of the imposed displacement is normalised at 0.1 macroscopic strain. The response to other loading can be easily recovered, since it is proportional to the loading at small strain.

"exotic" boundary conditions that the right lateral face is allowed to tilt, so that the panel can undergo any out of plane deformation without restrictions.

In addition, an equivalent homogeneous plate is considered with an effective behavior directly from the optimization and is included in the calculation. The rectangular plate is meshed with $50 \times 40$ discrete Kirchhoff triangular (DKT) shell elements. A sketch of the setting is provided in Figure 5(b). The right part is loaded in displacement along the direction $\boldsymbol{e}_{1}$ (resp. along direction $\boldsymbol{e}_{2}$ ), while all the other components and rotations are left free. Rigid body movements are eliminated by fixing the displacements and rotations on a node at the bottom left corner.

All calculations are conducted on the finite element solver Cast3M.

\subsection{Example 1}

The targets of the first microstructure to be optimised are given in the left side of Table 1. From a rapid analysis of these prescribed coefficients, it is clear that: the desired in-plane behavior should be auxetic (i.e., exhibit an in-plane effective negative Poisson's ratio of $v^{\text {target }}=-0.5$ ); The structure should exhibit a longitudinal extension-transverse bending coupled response, expressed by $B_{1122}^{\text {target }}$ and $B_{2211}^{\text {target }}$; A prescribed stiff longitudinal bending behavior, expressed by $D_{1111}^{\text {target }}$ and $D_{2222}^{\text {target }}$. To facilitate obtaining a desired quadratic symmetry, a symmetry of the shape is enforced along both the $O x z$ and $O y z$ planes, by symmetrizing the level set function during the algorithmic iterations. Additionally, the material volume fraction was constrained to be between $0.3 \leq|S| \leq 0.5$. The initial shape, depicted in Figure 4(a), is consisting of a square pattern of "cylindrical" micro-perforations, with a initial material volume fraction of $|S|=50 \%$.

\begin{tabular}{|c|c|c|c|c|c|c|c|c|c|c|c|}
\hline \multicolumn{6}{|c|}{$C^{\text {target }}$} & \multicolumn{6}{|c|}{$C^{*}$} \\
\hline 0.12 & -0.06 & $\star$ & $\star$ & $2.3 \mathrm{e}^{-3}$ & $\star$ & 0.120 & -0.059 & 0 & $-1.5 \mathrm{e}^{-3}$ & $1.8 \mathrm{e}^{-3}$ & 0 \\
\hline-0.06 & 0.12 & $\star$ & $2.3 \mathrm{e}^{-3}$ & $\star$ & $\star$ & -0.059 & 0.119 & 0 & $1.7 \mathrm{e}^{-3}$ & $1.0 \mathrm{e}^{-4}$ & 0 \\
\hline$\star$ & $\star$ & $\star$ & $\star$ & $\star$ & $\star$ & 0 & 0 & 0.03 & 0 & 0 & $-2.7 \mathrm{e}^{-4}$ \\
\hline$\star$ & $2.3 \mathrm{e}^{-3}$ & $\star$ & $6.3 e^{-4}$ & $\star$ & $\star$ & $-1.5 \mathrm{e}^{-3}$ & $1.7 \mathrm{e}^{-3}$ & 0 & $6.1 \mathrm{e}^{-4}$ & $-3.2 \mathrm{e}^{-5}$ & 0 \\
\hline $2.3 e^{-3}$ & $\star$ & $\star$ & $\star$ & $6.3 \mathrm{e}^{-4}$ & $\star$ & $1.8 \mathrm{e}^{-3}$ & $1.0 \mathrm{e}^{-4}$ & 0 & $-3.2 \mathrm{e}^{-5}$ & $6.0 \mathrm{e}^{-4}$ & 0 \\
\hline$\star$ & $\star$ & $\star$ & $\star$ & $\star$ & $\star$ & 0 & 0 & $-2.7 \mathrm{e}^{-4}$ & 0 & 0 & $1.7 \mathrm{e}^{-4}$ \\
\hline
\end{tabular}

Table 1: Values of the target stiffness tensors and the homogenised tensors for the final form of the microstructure in Figure 7. Only the entries that have numerical values in $\boldsymbol{C}^{\text {target }}$ were controlled. The remaining entries were left free.

The convergence history of the cost functional and of the volume constraint displayed in Figure 6(a) shows that the effective coefficients got stabilised in slightly more than 40 iterations and that the later iteration contributed only to small improvements without bringing the cost functional to less than $2 \times 10^{-4}$. We observe a significant drop in 


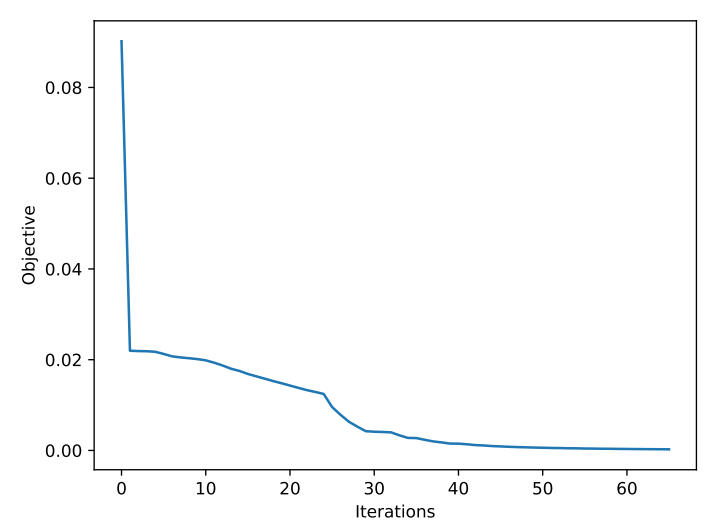

(a)

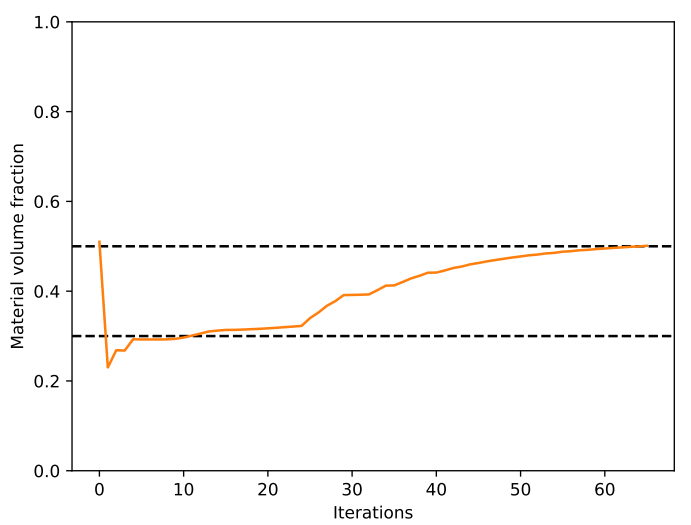

(b)

Figure 6: Evolution of the cost functional (a) and the volume constraint (b) with the number of iterations for the microstructure depicted in Figure 7. After 40 iterations we seem to have rather stable convergence both for the cost functional and volume constraint. The algorithm stops after 65 iterations, because the time step in the advection equation becomes too small.

the cost functional (Figure 6(a)) around iteration 25-30 that is associated with major changes in the microstructure's topology (see Movie 1 in the supplementary materials). Beyond this point, most of the changes contribute as minor improvements. The evolution of material volume fraction displayed in Figure 6(b) features an initial steep decrease down to 0.25 , attributed to the initial swelling of the holes, followed by a slower evolution to up to 0.5 , which is the upper limit of the proposed range of the constraint.

The final shape, depicted in Figure 7, is a lattice of complex shape. A view of a periodically assembled panel is also provided in Figure 8(a). The collected values of all the coefficients are included succinctly in the right side of Table 1. Let us now compare the values of $\boldsymbol{C}^{\text {target }}$ and $\boldsymbol{C}^{*}$. As prescribed, the final shape features an in-plane behavior with an effective Poisson's ratio of -0.5 . It exhibits significant values of $B_{1122}^{*}$ and $B_{2211}^{*}$, which come close to the target values. The longitudinal out of the plane behavior expressed through $D_{1111}^{*}$ and $D_{2222}^{*}$ also come close to the target value. Let us finally note that the panel will also exhibit a significant coupled shear response, expressed through $B_{1212}^{*}$. Complementary information on the plate deformation kinematics can be retrieved from the compliance tensor $S^{*}=\left(C^{*}\right)^{-1}$, provided in eq. (D.1) of Appendix D. The values in each row permit to assess the deformation modes for a given elementary load and indicate directly the final deformed shape.

Numerical simulations performed on a periodic panel loaded in the direction $O x$ reveal that the deformed shape in the linear regime will exhibit a significant negative Gaussian curvature, i.e., the panel will morph into a saddle shape (see Figure 8(b,e)). Conversely, when the panel is loaded in the direction $O y$, the deformed shape will morph into a circular dome (see Figure 8(c,f)).

The computational time needed to design Example 1 using the $100 \times 100 \times 25$ elements structured mesh took more 10 hours to run 65 iterations on a machine with $128 \mathrm{CPU}$. In order to reduce this computational time, we explore the relevance of an optimization with a coarser mesh, i.e. $50 \times 50 \times 12$ elements, which results in computational time between 0.5 to 2 hours (when 200 iterations are required). The following examples are obtained on this coarser mesh.

\subsection{Example 2}

The second microstructure to be optimised was obtained using the same initial parameters as in Example 1, except for the mesh which is made of $50 \times 50 \times 12$ elements. The targets of are recalled in the left side of Table 2 . Like before, a symmetry of the shape is enforced along both the $O x z$ and $O y z$ planes, by symmetrizing the level set function during the algorithmic iterations. The material volume fraction remains constrained to be between $0.3 \leq|S| \leq 0.5$. The other main difference is the considered initial guess. Here, the initial shape, depicted in Figure 4(e), is consisting of a regular "bubble" pattern, with an initial material volume fraction of $|S|=75 \%$. 


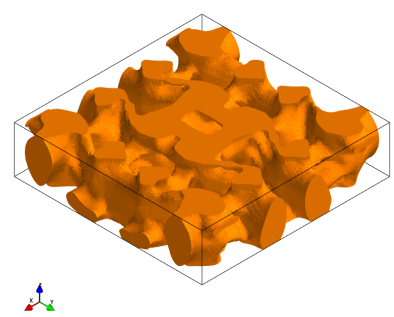

(a)

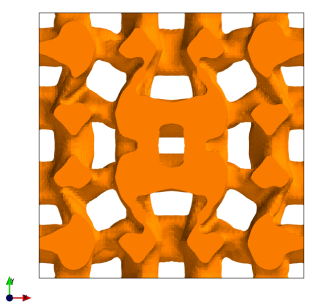

(b)

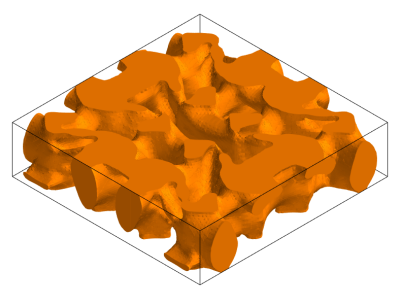

$\Delta p^{\circ}$

(c)

Figure 7: Optimally designed periodic panels accounting for extension-bending coupling effects, with an attained volume fraction of 0.5. Images (a) and (c) show the front and rear isometric view of the periodic cell, while image (b) shows a bird's eye view of the cell.

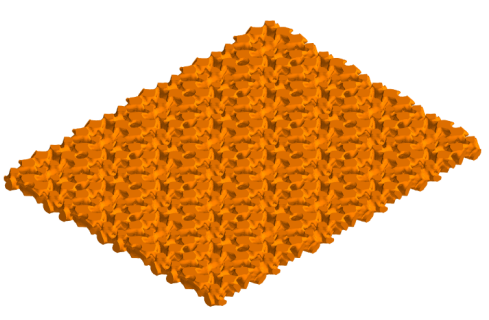

$x^{\prime}$

(a)

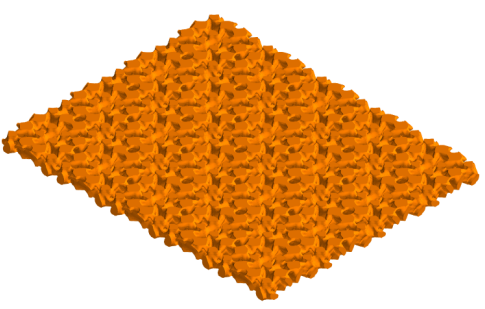

$x^{4}$

(d)

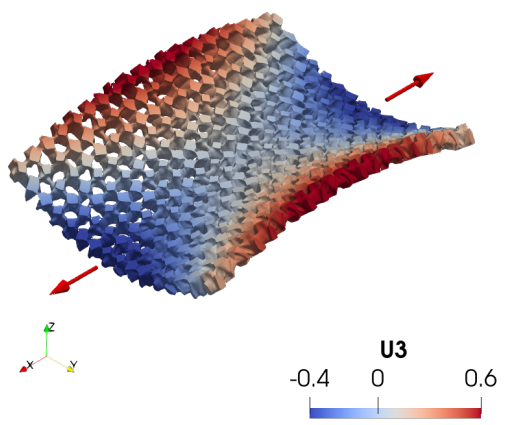

(b)

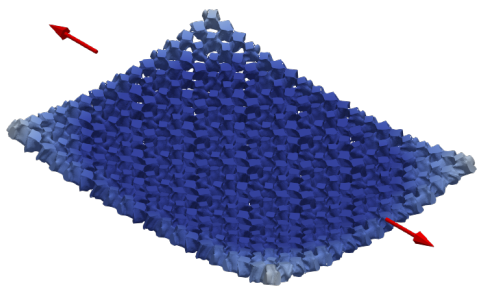

$\int^{x}$

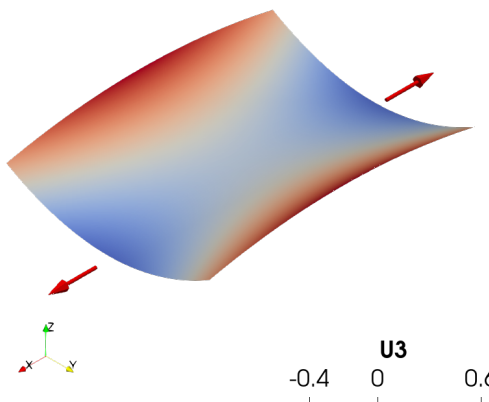

(c)

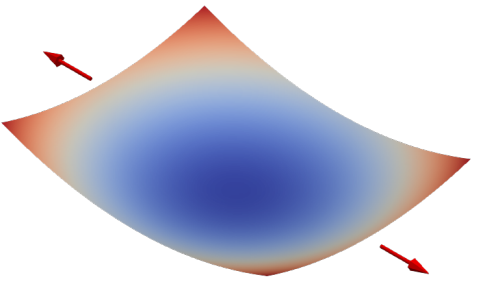

U3 0.9

(f)

Figure 8: $4 \times 5$ periodically assembled panel from the shape of Figure 7. Image (a) corresponds to its reference flat configuration. The panel is submitted to an uniaxial tensile load along $O x$ up to $10 \%$ macroscopic strain. Image (b) depicts the deformed state computed on the complete panel while image (c) shows its macroscopic response assuming the homogeneous equivalent plate model with the coefficients of Table 1 . The observed deformed shape is a saddle. The out-of-plane displacement is plotted as a color map on the deformed panel. It is recalled that the simulation was conducted in small strain, hence the out of plane response is proportional to the in-plane loading. The boundary conditions for the image (b) and (c) are reported in Figure 5. 


$\left[\begin{array}{ccc|ccc}\multicolumn{10}{c}{\boldsymbol{C}^{\text {target }}} & \multicolumn{10}{c}{\boldsymbol{C}^{*}} \\ -0.12 & -0.06 & \star & \star & 2.3 \mathrm{e}^{-3} & \star \\ \star & 0.12 & \star & 2.3 \mathrm{e}^{-3} & \star & \star \\ \hdashline & \star & \star & \star & \star & \star \\ \hline \star & 2.3 \mathrm{e}^{-3} & \star & 6.3 \mathrm{e}^{-4} & \star & \star \\ 2.3 \mathrm{e}^{-3} & \star & \star & \star & 6.3 \mathrm{e}^{-4} & \star \\ \star & \star & \star & \star & \star & \star\end{array}\right] \quad\left[\begin{array}{ccc|ccc}0.097 & -0.033 & 0 & 2.9 \mathrm{e}^{-4} & 2.2 \mathrm{e}^{-4} & 0 \\ -0.033 & 0.098 & 0 & 2.7 \mathrm{e}^{-4} & 2.8 \mathrm{e}^{-4} & 0 \\ 0 & 0 & 0.023 & 0 & 0 & 1.8 \mathrm{e}^{-4} \\ \hline 2.9 \mathrm{e}^{-4} & 2.7 \mathrm{e}^{-4} & 0 & 2.7 \mathrm{e}^{-4} & 6.2 \mathrm{e}^{-5} & 0 \\ 2.2 \mathrm{e}^{-4} & 2.8 \mathrm{e}^{-4} & 0 & 6.2 \mathrm{e}^{-5} & 2.7 \mathrm{e}^{-4} & 0 \\ 0 & 0 & 1.8 \mathrm{e}^{-4} & 0 & 0 & 2.0 \mathrm{e}^{-4}\end{array}\right]$

Table 2: Values of the target stiffness tensors and the homogenised tensors for the final form of the microstructure in Figure 10. Only the entries that have numerical values in $\boldsymbol{C}^{\text {target }}$ were controlled. The remaining entries were left free.

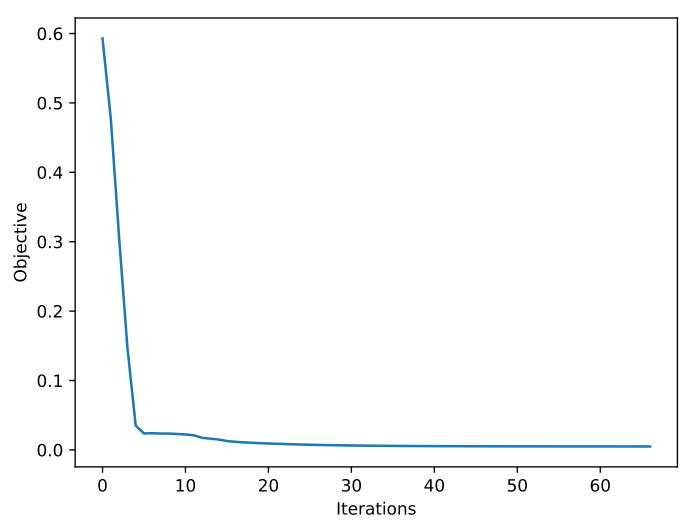

(a)

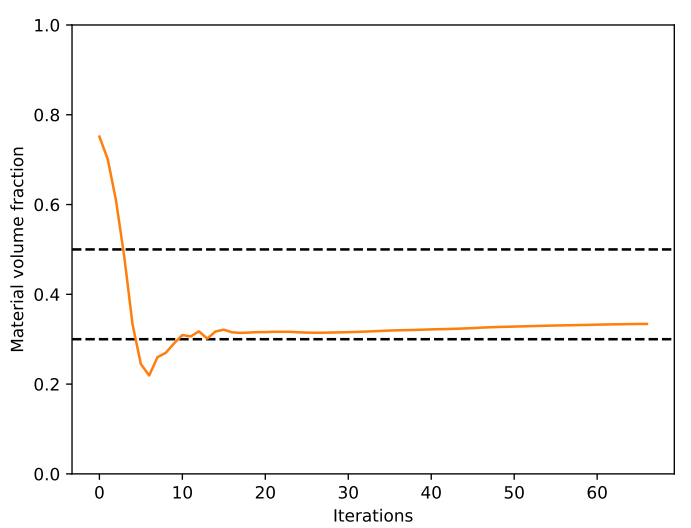

(b)

Figure 9: Evolution of the cost functional (a) and the volume constraint (b) with the number of iterations for the microstructure depicted in Figure 10. After 6 iterations, we seem to have rather stable convergence both for the cost functional and volume constraint. The algorithm stops after 65 iterations, because the time step in the advection equation becomes too small.

The convergence history of the cost functional and of the volume constraint displayed in Figure 9(a) shows that the objective gets stabilised in the very first 5 iterations, while the later iteration contributed only to small improvements without bringing the cost functional to less than $5 \times 10^{-3}$. Major changes in the microstructure's topology are observable until iteration 25 (see Movie 2 in the supplementary materials). Beyond this point, most of the changes contribute as minor improvements. Although the gain in the cost functional gets decreased by a factor of $10^{3}$, a remaining gap with respect to the target moduli can be read from Table 2, in particular in the sub-matrix $\boldsymbol{B}$. We conclude that this shape corresponds to a local minima for the objective function, but not as effective as the one in Table 1 . The evolution of material volume fraction displayed in Figure 9(b) features an initial steep decrease down to 0.25, attributed to the initial swelling of the holes, followed by a slower evolution to up to 0.33 .

The final shape, depicted in Figure 10, can be characterised as a "dimpled" sheet structure and looks similar to the designs imagined in [68]. A view of a periodically assembled panel is also provided in Figure 11(a). Although one might suspect that the structure exhibits further symmetries from the illustration in Figure 10(b), analyses of the volume fraction revealed that the structure is in fact slightly asymmetric along the direction $O z$ : the material volume fraction between $z \in[-h / 2,0]$ is equal to 0.35 , while it is equal to 0.31 for $z \in[0, h / 2]$. This asymmetry in the design is fundamental to obtain a significant coupling tensor $\boldsymbol{B}^{*}$. The collected values of all the coefficients of the aforementioned shape are included succinctly in the right side of Table 2. As prescribed, the final shape features an in-plane auxetic behavior, but the Poisson's ratio is of $v^{*}=-0.33$ rather than -0.5 . It exhibits a rather mild longitudinal extension-transverse bending coupling, with values for $B_{1122}^{*}$ and $B_{2211}^{*}$ being one order below the target value. Yet, noticing that the diagonal coefficient $B_{1111}^{*}$ and $B_{2222}^{*}$ are not negligible, meaning that the shape will feature a longitudinal extension-bending coupling. Complementary information on the plate deformation kinematics can be retrieved from the compliance tensor $S^{*}=\left(C^{*}\right)^{-1}$, provided in eq. (D.2) of Appendix D. 


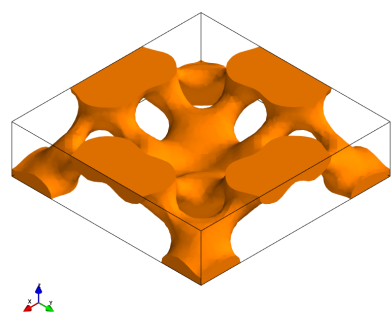

(a)

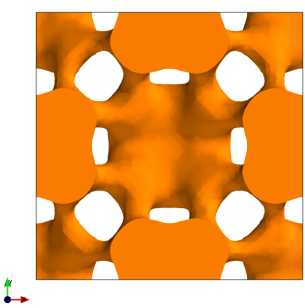

(b)

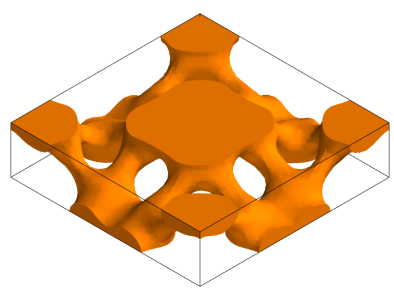

(c)

Figure 10: Optimally designed periodic panel resulting in a "dimpled" sheet structure. The attained volume fraction is $33 \%$. Images (a) and (c) show the top and bottom of the periodic cell, while image (b) shows a bird's eye view of the cell.

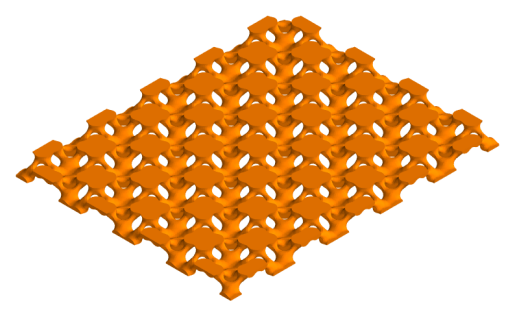

$x^{4}$

(a)

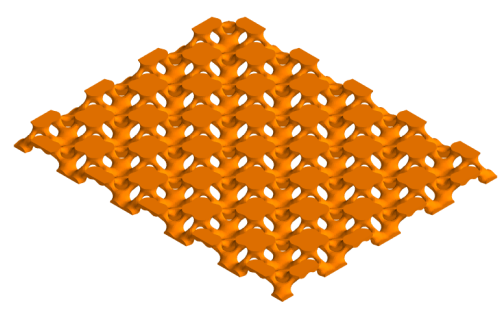

(x)

(d)

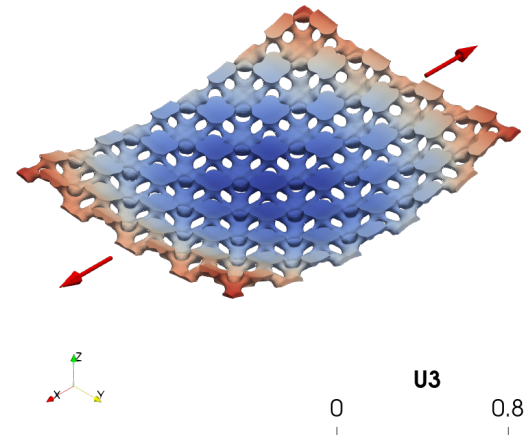

(b)

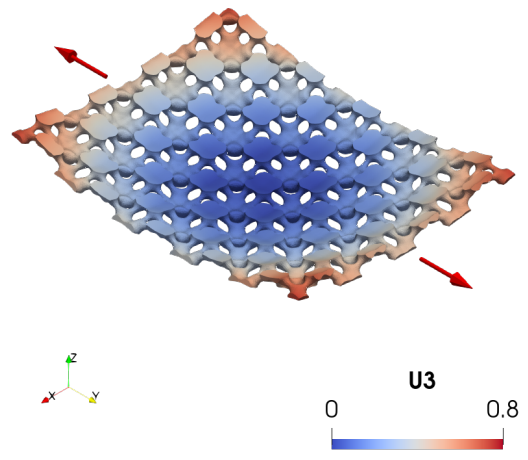

(e)

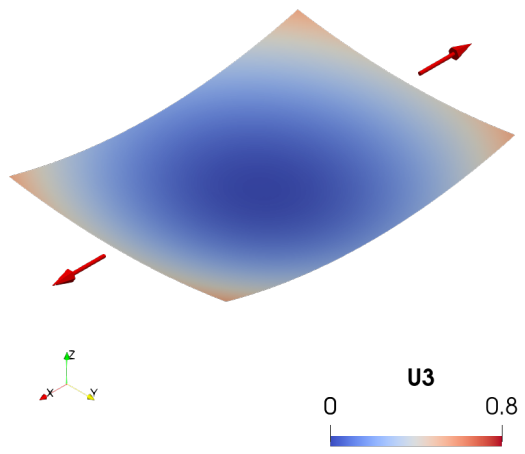

(c)

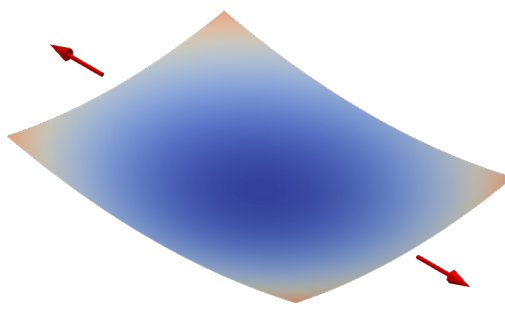

U3

0 0.8

(f)

Figure 11: $4 \times 5$ periodically assembled panel from the shape of Figure 10. Image (a) corresponds to its reference flat configuration. The panel is submitted to an uniaxial tensile load along $O x$ up to $10 \%$ macroscopic strain. Image (b) depicts the deformed state computed on the complete panel while image (c) shows its macroscopic response assuming the homogeneous equivalent plate model with the coefficients of Table 2 . The observed deformed shape is a saddle. The out-of-plane displacement is plotted as a color map on the deformed panel. It is recalled that the simulation was conducted in small strain, hence the out of plane response is proportional to the in-plane loading. The boundary conditions for the image (b) and (c) are reported in Figure 5. 
Numerical simulations performed on a periodic panel reveal that the deformed shape in the linear regime will exhibit a mild positive Gaussian curvature, i.e., the panel with morph into a quasi-circular dome shape (see Figure 11(b,e) for a loading in the $O x$ direction, and Figure 11(c,f) for a loading in the $O y$ direction). This result was anticipated, since the longitudinal components and the transverse components of $\boldsymbol{B}$ have similar positive values.

\subsection{Example 3}

The third microstructure to be optimised was obtained using the same initial parameters as in Example 1 and 2 . The targets of are recalled in the left side of Table 3. Like before, a symmetry of the shape is enforced along both the $O x z$ and $O y z$ planes, by symmetrizing the level set function during the algorithmic iterations. The material volume fraction remains constrained to be between $0.3 \leq|S| \leq 0.5$. A third initial guess is tested in this example. Here, the initial shape, depicted in Figure 4(d), consists of a diagonal pattern of "cone" micro-perforations, with an initial material volume fraction of $|S|=75 \%$.

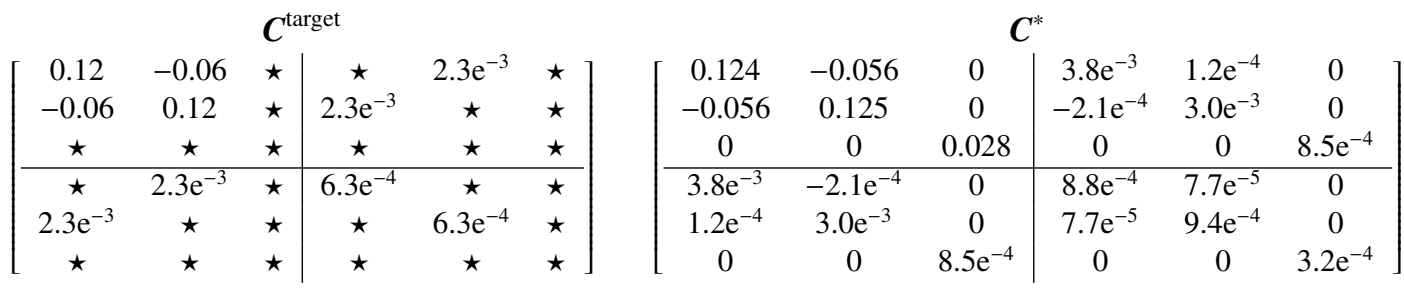

Table 3: Values of the target stiffness tensors and the homogenised tensors for the final form of the microstructure in Figure 13. Only the entries that have numerical values were controlled. The remaining entries were left free.

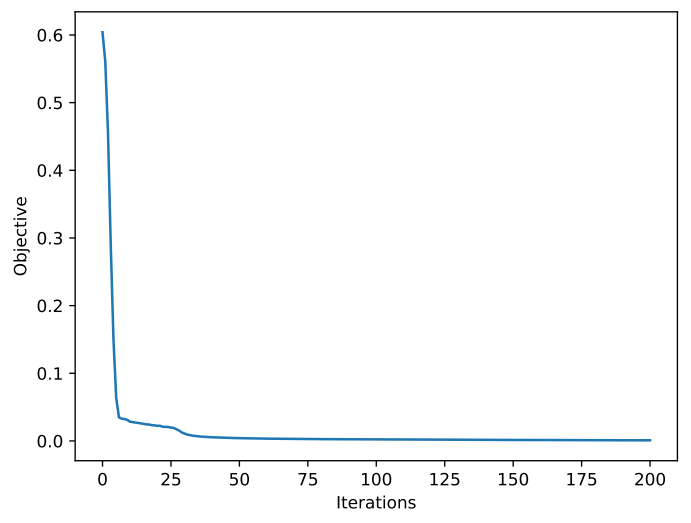

(a)

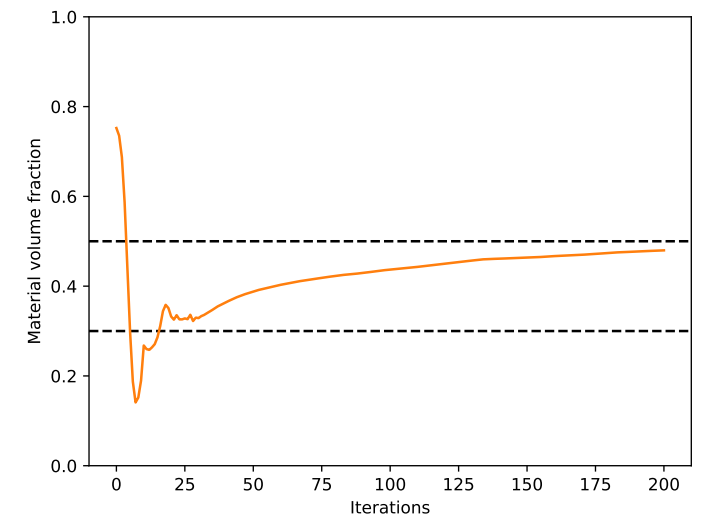

(b)

Figure 12: Evolution of the cost functional (a) and the volume constraint (b) for the number of iterations for the microstructure depicted in Figure 13. After 40 iterations, we seem to have rather stable convergence both for the cost functional and volume constraint. The algorithm stops after 200 iterations.

The convergence history of the cost functional and of the volume constraint displayed in Figure 12(a) shows that the shape gets stabilised after the first 30 iterations, while the later iteration contributed only to small improvements without bringing the cost functional to less than $10^{-3}$. This is corroborated by the evolution in the microstructure's topology observable until iteration 30 (see Movie 3 in the supplementary materials). Although the gain in the cost functional gets decreased by a factor of $10^{4}$, a remaining gap with respect to the target moduli can be read from Table 3, in particular in the sub-matrix $\boldsymbol{B}$. We conclude that this shape corresponds to a local minima for the objective function, but the shape is not as effective as the one in Table 1. The evolution of material volume fraction displayed in 


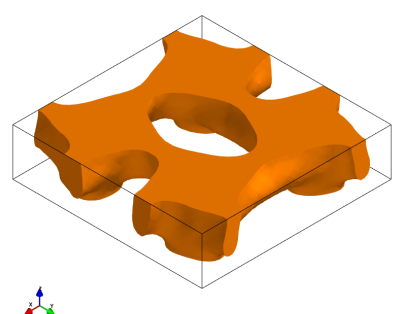

(a)

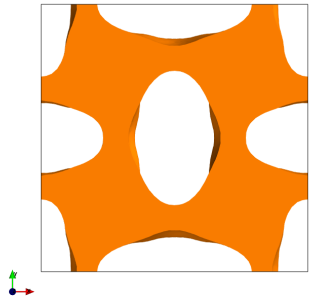

(b)

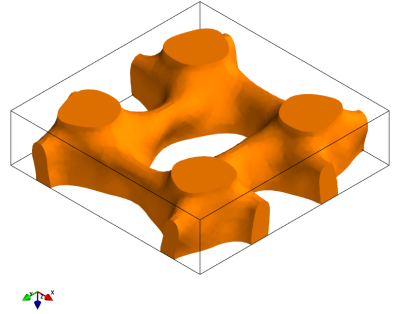

(c)

Figure 13: Optimally designed periodic panels accounting for bending-stretch effects. The attained volume fraction is 0.48 , which corresponds to lower bound of the volume interval set. Images (a) and (c) show the top and bottom of the periodic cell, while image (b) shows a bird's eye view of the cell.

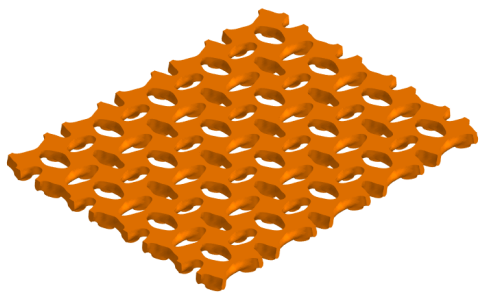

s.t.

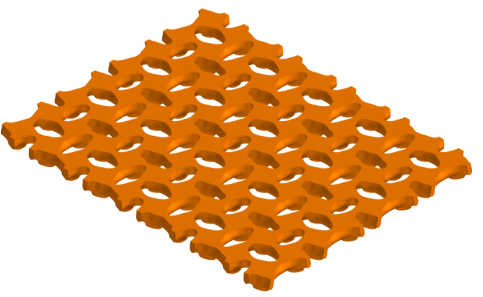

stis

(a)

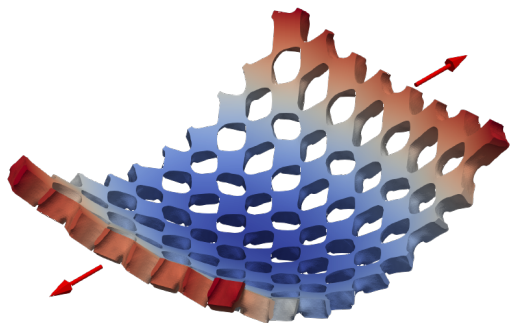

$e^{2}+2$

0

(b)

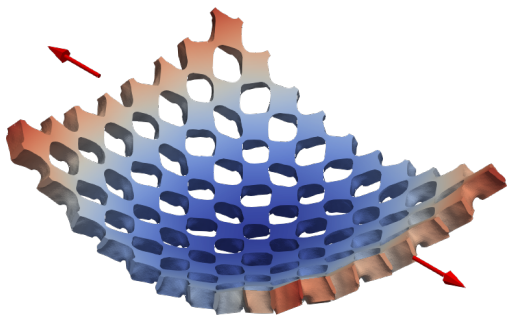

$\int^{2}+2$
U3

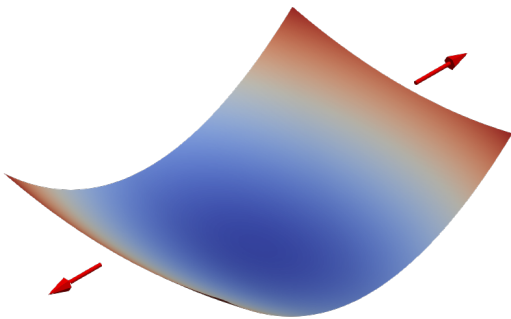

U3

(c)

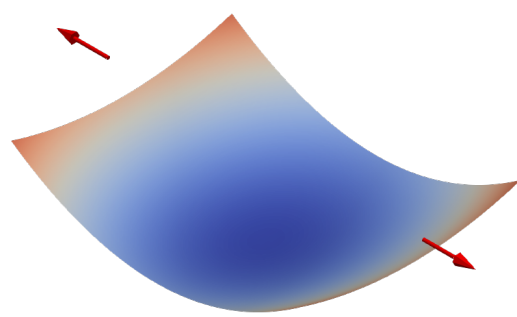

U3

(d)

(e)

(f)

Figure 14: $4 \times 5$ periodically assembled panel from the shape of Figure 13. Image (a) corresponds to its reference flat configuration. The panel is submitted to an uniaxial tensile load along $O x$ up to $10 \%$ macroscopic strain. Image (b) depicts the deformed state computed on the complete panel while image (c) shows its macroscopic response assuming the homogeneous equivalent plate model with the coefficients of Table 3 . The observed deformed shape is a saddle. The out-of-plane displacement is plotted as a color map on the deformed panel. It is recalled that the simulation was conducted in small strain, hence the out of plane response is proportional to the in-plane loading. The boundary conditions for the image (b) and (c) are reported in Figure 5. 
Figure 12(b) features an initial steep decrease down to 0.15, attributed to the initial swelling of the holes (Movie 3), followed by a and a slower evolution starting from iteration 40 to up to 0.48 .

As mentionned in the last paragraph of section 4, this example illustrates how two initial guesses can lead to two completely different final shapes despite the identical target. Here, the final shape, depicted in Figure 13, is of the rotating units type, discussed in [69] (see also the third example of a 2D design in [46]). A view of a periodically assembled panel is also provided in Figure 14(a). Again, a clear asymmetry in the design is visible especially at the hinges connecting the rotating blocks. The collected values of all the coefficients of the aforementioned shape are included succinctly in Table 3 . As prescribed, the resulting structure exhibits a "quadratic" symmetry. The final shape features an in-plane behavior with an effective Poisson's ratio of $v^{*}=-0.45$ which comes relatively close to the target. Like in Example 2, the obtained longitudinal extension-transverse bending coupling is quite small, with values for $B_{1122}^{*}$ and $B_{2211}^{*}$ being one order below the target value. By opposition with the last case, the longitudinal coupling coefficients $B_{1111}^{*}$ and $B_{2222}^{*}$ are one order of magnitude bigger than the transverse components $B_{1122}^{*}$ and $B_{2211}^{*}$. As a consequence, the obtained curvature will be elliptical, i.e., the curvature in the longitudinal direction will be stronger than the transverse one.

Numerical simulations performed on a periodic panel reveal that the deformed shape in the linear regime will exhibit a positive Gaussian curvature, i.e., the panel with morph into a elliptical dome shape (see Figure 14(b,e) for a loading in the $O x$ direction, and Figure 14(c,f) for a loading in the $O y$ direction).

\subsection{Example 4}

The targets of the last microstructure to be optimised are given in Table 4. The material volume fraction, once again, was constrained to be between $0.3 \leq|S| \leq 0.5$. The initial shape, depicted in Figure 4(d), consists of a diagonal pattern of "cone" micro-perforations. The collected values of all the coefficients of the aforementioned shape are succinctly included in Table 4.

$$
\left[\begin{array}{cccccc}
0.12 & -0.03 & \star & \star & 2.3 \mathrm{e}^{-3} & \star \\
-0.03 & 0.12 & \star & -2.3 \mathrm{e}^{-3} & \star & \star \\
\star & \star & \star & \star & \star & \star \\
\hline \star & -2.3 \mathrm{e}^{-3} & \star & 6.3 \mathrm{e}^{-4} & \star & \star \\
2.3 \mathrm{e}^{-3} & \star & \star & \star & 6.3 \mathrm{e}^{-4} & \star \\
\star & \star & \star & \star & \star & \star
\end{array}\right] \quad\left[\begin{array}{ccc|ccc}
0.106 & -2.8 \mathrm{e}^{-4} & 0 & 9.5 \mathrm{e}^{-3} & 1.8 \mathrm{e}^{-4} & 0 \\
-2.8 \mathrm{e}^{-4} & 0.114 & 0 & -8.3 \mathrm{e}^{-5} & -7.6 \mathrm{e}^{-3} & 0 \\
0 & 0 & 7.4 \mathrm{e}^{-3} & 0 & 0 & 1.0 \mathrm{e}^{-3} \\
\hline 9.5 \mathrm{e}^{-3} & -8.3 \mathrm{e}^{-5} & 0 & 9.4 \mathrm{e}^{-4} & 2.4 \mathrm{e}^{-5} & 0 \\
2.7 \mathrm{e}^{-4} & -7.6 \mathrm{e}^{-3} & 0 & 2.4 \mathrm{e}^{-5} & 7.3 e^{-4} & 0 \\
0 & 0 & 1.0 \mathrm{e}^{-3} & 0 & 0 & 1.1 \mathrm{e}^{-4}
\end{array}\right]
$$

Table 4: Values of the target stiffness tensors and the homogenised tensors for the final form of the microstructure in Figure 16. Only the entries that have numerical values were controlled. The remaining entries were left free.

The convergence history of the cost functional and of the volume constraint displayed in Figure 15(a) shows that the shape gets stabilised in the very first 10 iterations, while the later iteration contributed only to small improvements without bringing the cost functional to less than $6 \times 10^{-3}$. Although the gain in the cost functional gets decreased by a factor of $10^{3}$, a remaining gap with respect to the target moduli can be read from Table 4 , in particular in the block matrix $\boldsymbol{B}$. We conclude that this shape corresponds to a local minima for the objective function, but the shape is not as effective as the one in Table 1. The evolution of material volume fraction displayed in Figure 15(b) features an initial steep decrease down to 0.17 , attributed to the initial swelling of the holes, followed by an transient oscillatory evolution until iteration 20 and a slower evolution to up to 0.31 . The oscillations are also visible in the evolution in the microstructure's topology observable until iteration 20 (see Movie 4 in the supplementary materials).

The final shape is similar to the pantograph structures discussed in [70], however, we notice that the vertical beams are on top of the horizontal beams. The collected values of all the coefficients of the aforementioned shape are included succinctly in Table 4. The microstructure exhibits a mild auxetic response, but a remaining gap with respect to the target moduli can be read (significant difference in the $A_{1122}^{*}$ coefficient). It exhibits a rather small longitudinal extension-transverse bending coupling, with values for $B_{1122}^{*}$ and $B_{2211}^{*}$ being quite far to the target value. These remaining gaps are no surprise, given the fact that the code stopped after a little more than 40 iterations, much less than in the other examples. However, it is worth noticing that the diagonal coefficient $B_{1111}^{*}$ and $B_{2222}^{*}$ are huge, 


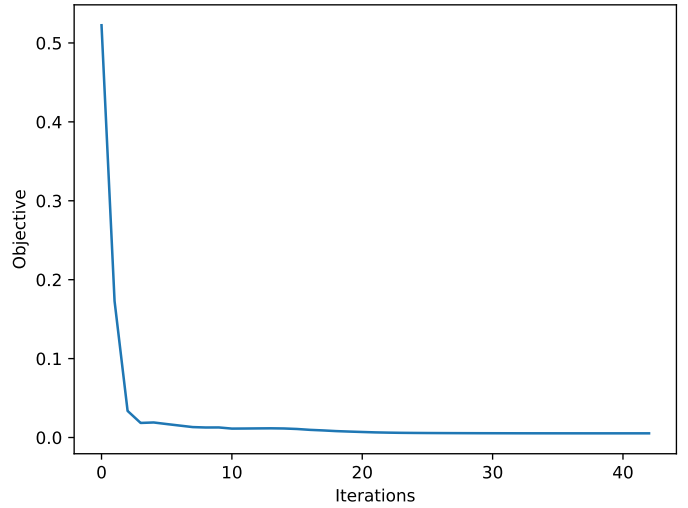

(a)

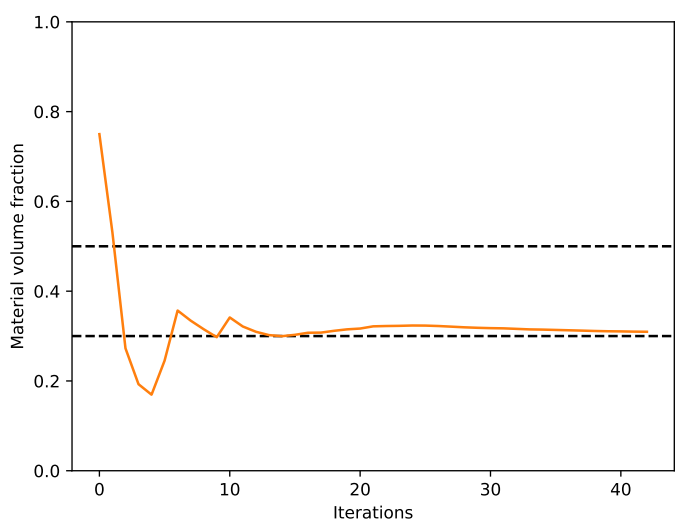

(b)

Figure 15: Evolution of the cost functional (a) and the volume constraint (b) for the number of iterations for the microstructure depicted in Figure 16. After 6 iterations, we seem to have rather stable convergence both for the cost functional and volume constraint. The algorithm stops after 43 iterations, because the time step in the advection equation becomes too small.

meaning that the shape features a strong longitudinal extension-bending coupling. Let us further note that the panel is almost decoupled in the shear response, expressed through a small value of $A_{1212}^{*}$. Finally, the longitudinal out of the plane behavior $D_{1111}^{*}$ and especially $D_{2222}^{*}$ come relatively close to the target value.

Numerical simulations performed on a periodic panel reveal that the deformed shape in the linear regime will morph into a cylinder (see Figure 17(b,e) for a loading in the $O x$ direction. The panel loaded in the $O y$ direction will also morph into a cylinder, but with the longitudinal curvature of opposite sign (see Figure 17(c,f)).

\section{Conclusion and perspectives}

We proposed a method for two-scale topology optimization of microstructured thin panels with in-plane periodicity. We use inverse homogenization and a level set method coupled with the Hadamard shape derivative to construct plate elastic moduli within the periodic cell in the context of the diffuse interphase approach that exhibit certain prescribed macroscopic behavior for a single material and "void" while simultaneously accounting for bending-stretching effects. By controlling the microstructure of the panel, we simultaneously controlled the in-plane, out-of-plane and their coupled behavior and in doing so we designed panels with an out-of-plane response that results in either a dome shaped structure or a saddle shaped structure under the action of in-plane loading. Interestingly, the direction of the loading can affect the type of obtained three-dimensional shape. By and large, these building blocks can be leveraged in systematic design of shape morphing structures. Moreover, the obtained shapes are directly realizable through additive manufacturing techniques.

\section{Acknowledgements}

F. A. acknowledges the support of the French doctoral fellowship "Contrat Doctoral Spécifique pour Normalien”. G. N. gratefully acknowledges the funding by the Deutsche Forschungsgemeinschaft (DFG, German Research Foundation) under Germany's Excellence Strategy - The Berlin Mathematics Research Center MATH+ (EXC-2046/1, project ID: 390685689) in project AA2-10. The authors are indebted to Grégoire Allaire, Charles Dapogny and Bogdan Vernescu for their help and fruitful discussions. 


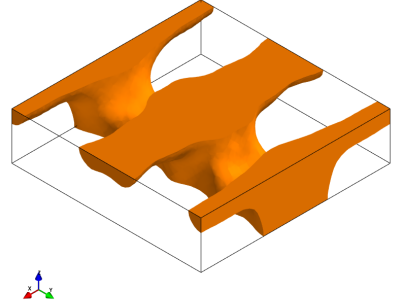

(a)

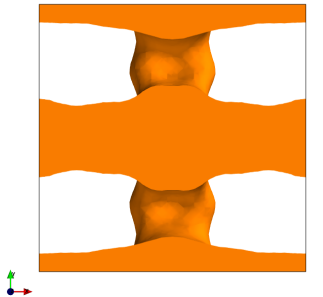

(b)

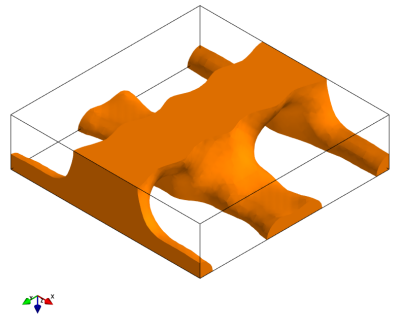

(c)

Figure 16: Optimally designed periodic panels accounting for bending-stretch effects mimicking a pantograph structure. The attained volume fraction is $30 \%$, which corresponds to lower bound of the volume interval set. Images (a) and (c) show the top and bottom of the periodic cell, while image (b) shows a bird's eye view of the cell.

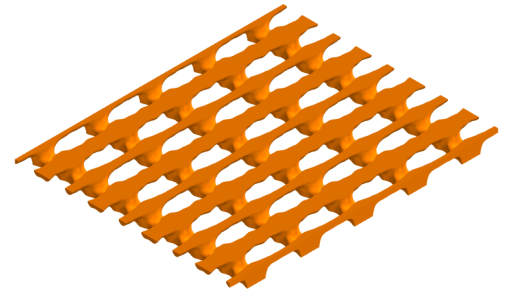

is

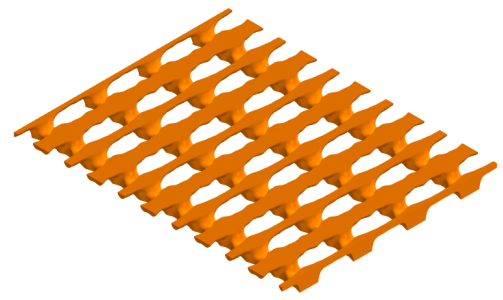

$s^{2}$

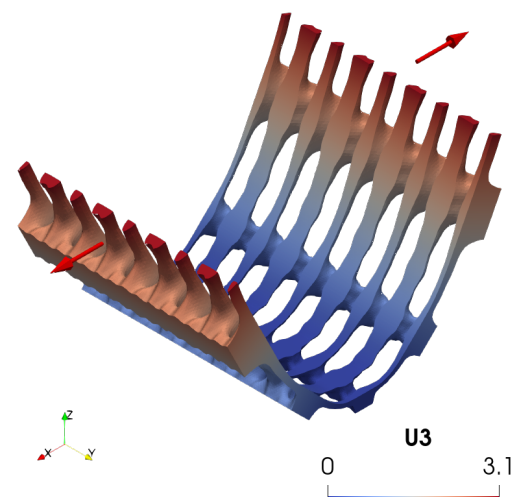

(b)

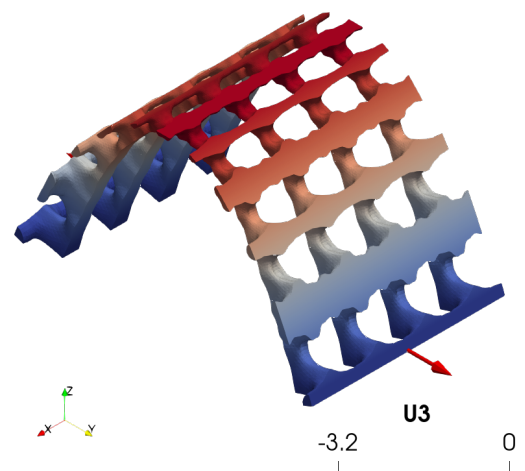

(e)

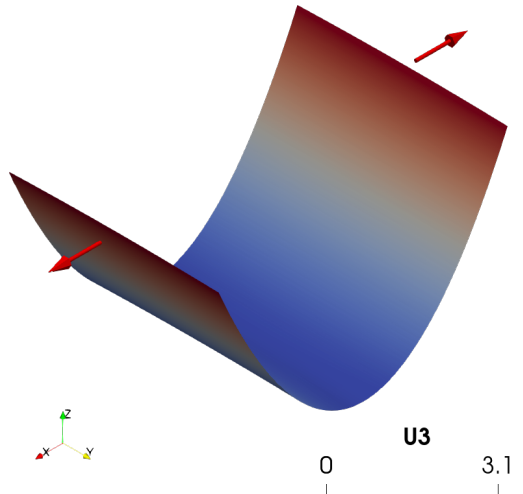

(c)

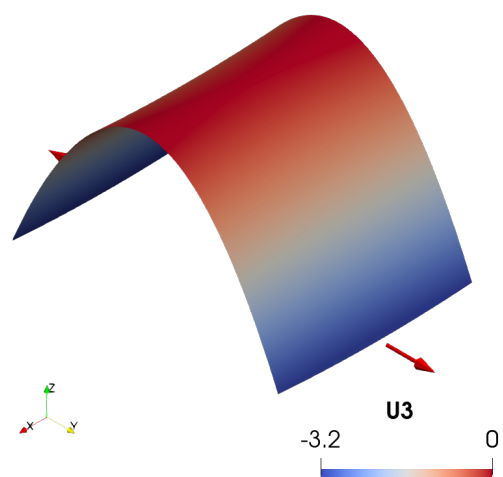

(f)

Figure 17: $4 \times 5$ periodically assembled panel from the shape of Figure 16. Image (a) corresponds to its reference flat configuration. The panel is submitted to an uniaxial tensile load along $O x$ up to $10 \%$ macroscopic strain. Image (b) depicts the deformed state computed on the complete panel while image (c) shows its macroscopic response assuming the homogeneous equivalent plate model with the coefficients of Table 4 . The observed deformed shape is a saddle. The out-of-plane displacement is plotted as a color map on the deformed panel. It is recalled that the simulation was conducted in small strain, hence the out of plane response is proportional to the in-plane loading. The boundary conditions for the image (b) and (c) are reported in Figure 5. 


\section{Supplementary material}

Supplementary material is available for this paper.

- Movie 1: Shape propagation of example 1;

- Movie 2: Shape propagation of example 2;

- Movie 3: Shape propagation of example 3;

- Movie 4: Shape propagation of example 4;

\section{References}

[1] T. Bückmann, N. Stenger, M. Kadic, J. Kaschke, A. Frölich, T. Kennerknecht, C. Eberl, M. Thiel, M. Wegener, Tailored 3D mechanical metamaterials made by dip-in direct-laser-writing optical lithography, Adv. Mater. 24 (20) (2012) 2710-2714. doi:10.1002/adma.201200584.

[2] F. Fraternali, A. Amendola, Mechanical modeling of innovative metamaterials alternating pentamode lattices and confinement plates, J. Mech. Phys. Solids 99 (2017) 259-271. doi:10.1016/j.jmps.2016.11.010.

[3] T. Frenzel, M. Kadic, M. Wegener, Three-dimensional mechanical metamaterials with a twist, Science 358 (6366) (2017) 1072-1074. doi : 10.1126/science. aao4640.

[4] O. R. Bilal, R. Süsstrunk, C. Daraio, S. D. Huber, Intrinsically polar elastic metamaterials, Adv. Mater. 29 (26) (2017) 1700540. doi : 10.1002/adma. 201700540.

[5] S. Krödel, L. Li, A. Constantinescu, C. Daraio, Stress relaxation in polymeric microlattice materials, Mater. Des. 130 (2017) $433-441$. doi:10.1016/j.matdes.2017.05.060.

[6] M. N. Andersen, F. Wang, O. Sigmund, On the competition for ultimately stiff and strong architected materials, Mater. Des. 198 (2021) 109356. doi:10.1016/j.matdes. 2020.109356.

[7] L. J. Gibson, M. F. Ashby, Cellular Solids, Cambridge University Press, 1997. doi : 10.1017/cbo9781139878326.

[8] G. W. Milton, The Theory of Composites, Cambridge University Press, 2002. doi :10.1017/cbo9780511613357.

[9] L. J. Gibson, M. F. Ashby, G. S. Schajer, C. I. Robertson, The mechanics of two-dimensional cellular materials, Proceedings of the Royal Society A: Mathematical, Physical and Engineering Sciences 382 (1782) (1982) 25-42. doi : 10.1098/rspa.1982.0087.

[10] G. Imbalzano, P. Tran, T. D. Ngo, P. V. S. Lee, A numerical study of auxetic composite panels under blast loadings, Compos. Struct. 135 (2016) 339-352. doi:10.1016/j.compstruct.2015.09.038.

[11] X. F. Xu, P. Qiao, Homogenized elastic properties of honeycomb sandwich with skin effect, Int. J. Solids Struct. 39 (8) (2002) $2153-2188$. doi:10.1016/s0020-7683(02)00111-7.

[12] N. Triantafyllidis, M. W. Schraad, Onset of failure in aluminum honeycombs under general in-plane loading, J. Mech. Phys. Solids 46 (6) (1998) 1089-1124. doi:10.1016/s0022-5096(97)00060-4.

[13] H. X. Zhu, N. J. Mills, The in-plane non-linear compression of regular honeycombs, Int. J. Solids Struct. 37 (13) (2000) 1931-1949. doi : 10.1016/s0020-7683(98)00324-2.

[14] F. López Jiménez, N. Triantafyllidis, Buckling of rectangular and hexagonal honeycomb under combined axial compression and transverse shear, Int. J. Solids Struct. 50 (24) (2013) 3934-3946. doi:10.1016/j.ijsolstr.2013.08.001.

[15] K. Oliver, A. Seddon, R. S. Trask, Morphing in nature and beyond: a review of natural and synthetic shape-changing materials and mechanisms, J. Mater. Sci. 51 (24) (2016) 10663-10689. doi:10.1007/s10853-016-0295-8.

[16] R. Guseinov, E. Miguel, B. Bickel, CurveUps : Shaping objects from flat plates with tension-actuated curvature, ACM Trans. Graph. 36 (4) (2017) 1-12. doi:10.1145/3072959.3073709.

[17] L. Malomo, J. Pérez, E. Iarussi, N. Pietroni, E. Miguel, P. Cignoni, B. Bickel, Flexmaps: Computational design of flat flexible shells for shaping 3d objects, ACM Trans. Graph. 37 (6) (2018) 1-14. doi:10.1145/3272127.3275076.

[18] R. Guseinov, C. McMahan, J. Pérez, C. Daraio, B. Bickel, Programming temporal morphing of self-actuated shells, Nat. Commun. 11 (1) (1 2020). doi:10.1038/s41467-019-14015-2.

[19] A. Rafsanjani, D. Pasini, Bistable auxetic mechanical metamaterials inspired by ancient geometric motifs, Extreme Mech. Lett. 9 (2016) 291-296. doi:10.1016/j.eml.2016.09.001.

[20] A. Kotikian, C. McMahan, E. C. Davidson, J. M. Muhammad, R. D. Weeks, C. Daraio, J. A. Lewis, Untethered soft robotic matter with passive control of shape morphing and propulsion, Sci. Robot. 4 (33) (2019) eaax7044. doi:10.1126/scirobotics .aax7044.

[21] A. A. Bauhofer, S. Krödel, J. Rys, O. R. Bilal, A. Constantinescu, C. Daraio, Harnessing photochemical shrinkage in direct laser writing for shape morphing of polymer sheets, Adv. Mater. 29 (42) (2017) 1703024. doi :10.1002/adma. 201703024.

[22] T. Chen, O. R. Bilal, K. Shea, C. Daraio, Harnessing bistability for directional propulsion of soft, untethered robots, Proc. Natl. Acad. Sci. U.S.A. 115 (22) (2018) 5698-5702. doi:10.1073/pnas. 1800386115.

[23] G. Allaire, Shape Optimization by the Homogenization Method, Springer New York, 2002. doi : 10.1007/978-1-4684-9286-6.

[24] A. Bensoussan, J.-L. Lions, G. Papanicolaou, Asymptotic Analysis for Periodic Structures, North-Holland Pub. Co. Sole distributors for the U.S.A. and Canada, Elsevier North-Holland, Amsterdam New York New York, 1978. doi:10.1090/chel/374.

[25] E. Sanchez-Palencia, Non-Homogeneous Media and Vibration Theory, Springer Berlin Heidelberg, 1980. doi:10. 1007/3-540-10000-8.

[26] D. Cioranescu, P. Donato, An introduction to homogenization, Oxford University Press, Oxford New York, 1999. 
[27] N. S. Bakhvalov, G. Panasenko, Homogenisation: Averaging Processes in Periodic Media, Springer Netherlands, 2011. doi:10.1007/ 978-94-009-2247-1.

[28] C. C. Mei, B. Vernescu, Homogenization Methods for Multiscale Mechanics, World Scientific, 2010. doi:10.1142/7427.

[29] D. Caillerie, J.-C. Nedelec, Thin elastic and periodic plates, Math. Methods Appl. Sci. 6 (1) (1984) 159-191. doi:10.1002/mma. 1670060112

[30] R. V. Kohn, M. Vogelius, A new model for thin plates with rapidly varying thickness, Int. J. Solids Struct. 20 (4) (1984) 333-350. doi: 10.1016/0020-7683(84)90044-1.

[31] K. Sab, A. Lebée, Homogenization of Heterogeneous Thin and Thick Plates, John Wiley \& Sons, Inc., 2015. doi:10.1002/ 9781119005247

[32] H. A. Eschenauer, N. Olhoff, Topology optimization of continuum structures: A review*, Appl. Mech. Rev. 54 (4) (2001) 331-390. doi: $10.1115 / 1.1388075$

[33] M. P. Bendsøe, O. Sigmund, Topology Optimization, Springer Berlin Heidelberg, 2004. doi : 10.1007/978-3-662-05086-6.

[34] G. Allaire, Conception optimale de structures, Springer Berlin Heidelberg, 2007. doi : 10.1007/978-3-540-36856-4.

[35] M. P. Bendsøe, N. Kikuchi, Generating optimal topologies in structural design using a homogenization method, Comput. Methods Appl. Mech. Eng. 71 (2) (1988) 197-224. doi:10.1016/0045-7825(88)90086-2.

[36] G. Allaire, F. Jouve, A.-M. Toader, A level-set method for shape optimization, Comptes Rendus Math. 334 (12) (2002) 1125-1130. doi : 10.1016/s1631-073x(02)02412-3.

[37] M. P. Bendsøe, Optimal shape design as a material distribution problem, Struct. Optim. 1 (4) (1989) 193-202. doi:10.1007/bf01650949.

[38] M. Carraturo, E. Rocca, E. Bonetti, D. Hömberg, A. Reali, F. Auricchio, Graded-material design based on phase-field and topology optimization, Comput. Mech. 64 (6) (2019) 1589-1600. doi:10.1007/s00466-019-01736-w.

[39] F. Auricchio, E. Bonetti, M. Carraturo, D. Hömberg, A. Reali, E. Rocca, A phase-field-based graded-material topology optimization with stress constraint, Math. Models Methods Appl. Sci. 30 (08) (2020) 1461-1483. doi : 10.1142/s0218202520500281.

[40] G. Allaire, F. Jouve, A.-M. Toader, Structural optimization using sensitivity analysis and a level-set method, J. Comput. Phys. 194 (1) (2004) 363-393. doi:10.1016/j.jcp.2003.09.032.

[41] M. Y. Wang, X. Wang, D. Guo, A level set method for structural topology optimization, Comput. Methods Appl. Mech. Eng. 192 (1-2) (2003) 227-246. doi:10.1016/s0045-7825(02)00559-5.

[42] X. Wang, Y. Mei, M. Y. Wang, Level-set method for design of multi-phase elastic and thermoelastic materials, Int. J. Mech. Mater. Des. 1 (3) (2004) 213-239. doi:10.1007/s10999-005-0221-8.

[43] Y. Wang, Z. Luo, N. Zhang, Z. Kang, Topological shape optimization of microstructural metamaterials using a level set method, Comput. Mater. Sci. 87 (2014) 178-186. doi:10.1016/j.commatsci.2014.02.006.

[44] F. Wang, O. Sigmund, J. S. Jensen, Design of materials with prescribed nonlinear properties, J. Mech. Phys. Solids 69 (2014) 156-174. doi: $10.1016 / j \cdot j m p s .2014 .05 .003$.

[45] G. Nika, A. Constantinescu, Design of multi-layer materials using inverse homogenization and a level set method, Comput. Methods Appl. Mech. Eng. 346 (2019) 388-409. doi:10.1016/j.cma.2018.11.029.

[46] F. Agnelli, A. Constantinescu, G. Nika, Design and testing of 3d-printed micro-architectured polymer materials exhibiting a negative poisson's ratio, Contin. Mech. Thermodyn. 32 (2) (2020) 433-449. doi:10.1007/s00161-019-00851-6.

[47] J. Schwerdtfeger, F. Wein, G. Leugering, R. F. Singer, C. Körner, M. Stingl, F. Schury, Design of auxetic structures via mathematical optimization, Adv. Mater. 23 (22-23) (2011) 2650-2654. doi:10.1002/adma . 201004090.

[48] N. Aage, E. Andreassen, B. S. Lazarov, Topology optimization using PETSc: An easy-to-use, fully parallel, open source topology optimization framework, Struct. Multidiscip. Optim. 51 (3) (2014) 565-572. doi : 10.1007/s00158-014-1157-0.

[49] E. Andreassen, B. S. Lazarov, O. Sigmund, Design of manufacturable 3d extremal elastic microstructure, Mech. Mater. 69 (1) (2014) 1-10. doi: 10.1016/j.mechmat.2013.09.018.

[50] P. Vogiatzis, S. Chen, X. Wang, T. Li, L. Wang, Topology optimization of multi-material negative Poisson's ratio metamaterials using a reconciled level set method, Comput. Aided Des. 83 (2017) 15-32. doi:10.1016/j .cad. 2016.09.009.

[51] F. Wang, Systematic design of 3D auxetic lattice materials with programmable Poisson's ratio for finite strains, J. Mech. Phys. Solids 114 (2018) 303-318. doi:10.1016/j.jmps .2018.01.013.

[52] L. V. Gibiansky, A. V. Cherkaev, Design of composite plates of extremal rigidity, in: A. V. Cherkaev, R. V. Kohn (Eds.), Topics in the Mathematical Modelling of Composite Materials, Springer International Publishing, 1997, pp. 95-137. doi : 10.1007/978-3-319-97184-1_5.

[53] S. Nishi, K. Terada, J. Kato, S. Nishiwaki, K. Izui, Two-scale topology optimization for composite plates with in-plane periodicity, Int. J. Numer. Methods Eng. 113 (8) (2017) 1164-1188. doi:10.1002/nme.5545.

[54] F. Agnelli, M. Tricarico, A. Constantinescu, Shape-shifting panel from 3D printed undulated ribbon lattice, Extreme Mech. Lett. 42 (2021) 101089. doi: 10.1016/j.eml.2020.101089.

[55] G. Allaire, C. Dapogny, G. Delgado, G. Michailidis, Multi-phase structural optimization via a level set method, ESAIM: Control Optim. Calc. Var. 20 (2) (2014) 576-611. doi:10.1051/cocv/2013076.

[56] G. W. Milton, A. V. Cherkaev, Which elasticity tensors are realizable?, J. Eng. Mater. Technol. Trans. 117 (4) (1995) 483-493. doi : $10.1115 / 1.2804743$.

[57] G. W. Milton, R. V. Kohn, Variational bounds on the effective moduli of anisotropic composites, J. Mech. Phys. Solids 36 (6) (1988) 597-629. doi : 10.1016/0022-5096(88) 90001-4.

[58] O. Sigmund, On the usefulness of non-gradient approaches in topology optimization, Struct. Multidiscip. Optim. 43 (5) (2011) 589-596. doi:10.1007/s00158-011-0638-7.

[59] F. Murat, J. Simon, Etude de problemes d'optimal design, in: Lect. Notes Comput. Sci., Springer Berlin Heidelberg, 1976, pp. 54-62. doi : 10.1007/3-540-07623-9_279.

[60] O. Pironneau, Optimal Shape Design for Elliptic Systems, Springer Berlin Heidelberg, 1984. doi:10.1007/978-3-642-87722-3.

[61] M. C. Delfour, J.-P. Zolésio, Shapes and geometries: metrics, analysis, differential calculus, and optimization, Society for Industrial and Applied Mathematics, 2011. doi:10.1137/1.9780898719826. 
[62] S. J. Osher, J. A. Sethian, Fronts propagating with curvature-dependent speed: algorithms based on hamilton-jacobi formulations, J. Comput. Phys. 79 (1) (1988) 12-49. doi:10.1016/0021-9991(88)90002-2.

[63] D. Bertsekas, Constrained Optimization and Lagrange Multiplier Methods, Athena Scientific, Belmont, Mass, 1982. doi:10.1016/ c2013-0-10366-2.

[64] J. Nocedal, S. J. Wright, Numerical Optimization, Springer New York, 2006. doi : 10.1007/978-0-387-40065-5.

[65] G. Michailidis, Manufacturing constraints and multi-phase shape and topology optimization via a level-set method, phdthesis, Ecole polytechnique X (2014). URL https: //pastel.archives-ouvertes.fr/pastel-00937306

[66] T. T. C. Bui, C. Dapogny, P. Frey, An accurate anisotropic adaptation method for solving the level set advection equation, Int. J. Numer. Methods Fluids 70 (7) (2011) 899-922. doi :10.1002/fld.2730.

[67] C. Dapogny, P. Frey, Computation of the signed distance function to a discrete contour on adapted triangulation, Calcolo 49 (3) (2012) 193-219. doi: 10.1007/s10092-011-0051-z.

[68] B. Hassani, S. M. Tavakkoli, H. Ghasemnejad, Simultaneous shape and topology optimization of shell structures, Struct. Multidiscipl. Optim. 48 (1) (2013) 221-233. doi:10.1007/s00158-013-0894-9.

[69] J. N. Grima, V. Zammit, R. Gatt, A. Alderson, K. E. Evans, Auxetic behaviour from rotating semi-rigid units, Phys. Status Solidi B 244 (3) (2007) 866-882. doi:10.1002/pssb.200572706.

[70] F. Dell'Isola, P. Seppecher, M. Spagnuolo, E. Barchiesi, F. Hild, T. Lekszycki, I. Giorgio, L. Placidi, U. Andreaus, M. Cuomo, S. R. Eugster, A. Pfaff, K. Hoschke, R. Langkemper, E. Turco, R. Sarikaya, A. Misra, M. D. Angelo, F. D’Annibale, A. Bouterf, X. Pinelli, A. Misra, B. Desmorat, M. Pawlikowski, C. Dupuy, D. Scerrato, P. Peyre, M. Laudato, L. Manzari, P. Göransson, C. Hesch, S. Hesch, P. Franciosi, J. Dirrenberger, F. Maurin, Z. Vangelatos, C. Grigoropoulos, V. Melissinaki, M. Farsari, W. Muller, B. E. Abali, C. Liebold, G. Ganzosch, P. Harrison, R. Drobnicki, L. Igumnov, F. Alzahrani, T. Hayat, Advances in pantographic structures: design, manufacturing, models, experiments and image analyses, Contin. Mech. Thermodyn. 31 (4) (2019) 1231-1282. doi:10.1007/s00161-019-00806-x.

[71] J. Céa, Conception optimale ou identification de formes, calcul rapide de la dérivée directionnelle de la fonction coût, ESAIM: Math. Model Numer. Anal. 20 (3) (1986) 371-402. doi : 10.1051/m2an/1986200303711.

\section{Appendix A. Shape propagation analysis}

Shape derivative in the smoothed-interface context. Using the method of Céa, discussed in [71], for the calculation of the shape derivative of the objective function, we formulate the Lagrangian function $\mathcal{L}: W^{1, \infty}\left(Y, \mathbb{R}^{3}\right) \times \mathcal{V} \times \mathcal{V} \times$ $\mathcal{V} \times \mathcal{V} \rightarrow \mathbb{R}$ as follows:

$$
\begin{aligned}
\mathcal{L}\left(S, \boldsymbol{\xi}^{\gamma \delta}, \boldsymbol{\Xi}, \boldsymbol{\zeta}^{\gamma \delta}, \boldsymbol{Z}\right) & =\frac{1}{2} \eta_{\alpha \beta \gamma \delta}^{A}\left(\boldsymbol{A}_{\alpha \beta \gamma \delta}^{*}-\boldsymbol{A}_{\alpha \beta \gamma \delta}^{\mathrm{target}}\right)^{2}+\frac{1}{2} \eta_{\alpha \beta \gamma \delta}^{B}\left(\boldsymbol{B}_{\alpha \beta \gamma \delta}^{*}-\boldsymbol{B}_{\alpha \beta \gamma \delta}^{\mathrm{target}}\right)^{2}+\frac{1}{2} \eta_{\alpha \beta \gamma \delta}^{D}\left(\boldsymbol{D}_{\alpha \beta \gamma \delta}^{*}-\boldsymbol{D}_{\alpha \beta \gamma \delta}^{\mathrm{target}}\right)^{2} \\
& +\int_{Y} \boldsymbol{C}(\boldsymbol{y}):\left(\boldsymbol{E}^{\gamma \delta}+\boldsymbol{\varepsilon}_{\boldsymbol{y}}\left(\boldsymbol{\xi}^{\gamma \delta}\right)\right): \boldsymbol{\varepsilon}_{\boldsymbol{y}}(\boldsymbol{\Xi}) d \boldsymbol{y} \\
& +\int_{Y} \boldsymbol{C}(\boldsymbol{y}):\left(\boldsymbol{X}^{\alpha \beta}+\boldsymbol{\varepsilon}_{\boldsymbol{y}}\left(\boldsymbol{\zeta}^{\alpha \beta}\right)\right): \boldsymbol{\varepsilon}_{\boldsymbol{y}}(\boldsymbol{Z}) d \boldsymbol{y}
\end{aligned}
$$

Here, $\boldsymbol{\Xi}, \boldsymbol{Z}$ are intended as the Lagrange multipliers associated to the enforcement of the state equations. $\boldsymbol{\xi}^{\gamma \delta}, \boldsymbol{\Xi}, \boldsymbol{\zeta}^{\gamma \delta}$ and $\boldsymbol{Z}$ are vector-valued functions defined in $Y$, which do not depend on $S$. As usual, the stationarity of the Lagrangian provides the optimality conditions for the minimization problem.

Direct problem. Differentiating $\mathcal{L}$ in (A.1) with respect to $\Xi$ in the direction of a test function $\varphi \in H^{1}\left(Y, \mathbb{R}^{3}\right)$ gives:

$$
\left\langle\frac{\partial \mathcal{L}}{\partial \boldsymbol{\Xi}} \mid \varphi\right\rangle=\int_{Y} \boldsymbol{C}(\boldsymbol{y}):\left(\boldsymbol{E}^{\gamma \delta}+\boldsymbol{\varepsilon}_{y}\left(\xi^{\gamma \delta}\right)\right): \boldsymbol{\varepsilon}_{y}(\boldsymbol{\varphi}) d \boldsymbol{y}
$$

Upon setting the above equation equal to zero, we recover the variational formulation of first state equation (2.7). Similarly, differentiating $\mathcal{L}$ in (A.1) with respect to $\boldsymbol{Z}$ in the direction of a test function $\varphi \in H^{1}\left(Y, \mathbb{R}^{3}\right)$ gives:

$$
\left\langle\frac{\partial \mathcal{L}}{\partial \boldsymbol{Z}} \mid \varphi\right\rangle=\int_{Y} \boldsymbol{C}(\boldsymbol{y}):\left(\boldsymbol{X}^{\alpha \beta}+\boldsymbol{\varepsilon}_{y}\left(\zeta^{\alpha \beta}\right)\right): \boldsymbol{\varepsilon}_{y}(\varphi) d \boldsymbol{y}
$$

Upon setting the above equation equal to zero, we recover the variational formulation of second state equation (2.8). 
Adjoint problem. The partial derivative of $\mathcal{L}$ in (A.1) with respect to $\xi^{\gamma \delta}$ in the direction of a test function $\psi \in$ $H^{1}\left(Y, \mathbb{R}^{3}\right)$ results in:

$$
\begin{aligned}
\left\langle\frac{\partial \mathcal{L}}{\partial \boldsymbol{\xi}^{\gamma \delta}} \mid \boldsymbol{\psi}\right\rangle & =\frac{r}{|Y|} \eta_{\alpha \beta \gamma \delta}^{A}\left(\boldsymbol{A}_{\alpha \beta \gamma \delta}^{*}-\boldsymbol{A}_{\alpha \beta \gamma \delta}^{\mathrm{target}}\right) \int_{Y} \boldsymbol{C}(\boldsymbol{y}): \boldsymbol{\varepsilon}_{\boldsymbol{y}}(\boldsymbol{\psi}):\left(\boldsymbol{E}^{\gamma \delta}+\boldsymbol{\varepsilon}_{\boldsymbol{y}}\left(\boldsymbol{\xi}^{\gamma \delta}\right)\right) d \boldsymbol{y} \\
& +\frac{r}{|Y|} \eta_{\alpha \beta \gamma \delta}^{B}\left(\boldsymbol{B}_{\alpha \beta \gamma \delta}^{*}-\boldsymbol{B}_{\alpha \beta \gamma \delta}^{\mathrm{target}}\right) \int_{Y} \boldsymbol{C}(\boldsymbol{y}): \boldsymbol{\varepsilon}_{\boldsymbol{y}}(\boldsymbol{\psi}):\left(\boldsymbol{X}^{\gamma \delta}+\boldsymbol{\varepsilon}_{\boldsymbol{y}}\left(\boldsymbol{\zeta}^{\gamma \delta}\right)\right) d \boldsymbol{y} \\
& +\int_{Y} \boldsymbol{C}(\boldsymbol{y}): \boldsymbol{\varepsilon}_{\boldsymbol{y}}(\boldsymbol{\psi}): \boldsymbol{\varepsilon}_{\boldsymbol{y}}(\boldsymbol{\Xi}) d \boldsymbol{y}
\end{aligned}
$$

The integral over $Y$ on the first two lines is equal to 0 from the state equations (2.7) and (2.8). Moreover, by choosing $\psi=\boldsymbol{\Xi}$ and using the positive definiteness of $\boldsymbol{C}$ as well as the $Y$-periodicity of $\boldsymbol{\Xi}$, we obtain that the solution of the adjoint state is identically zero, $\boldsymbol{\Xi}=\mathbf{0}$. Similarly, the partial derivative of $\mathcal{L}$ with respect to $\zeta^{\gamma \delta}$ in the direction of a test function $\psi \in H^{1}\left(Y, \mathbb{R}^{3}\right)$ results in:

$$
\begin{aligned}
\left\langle\frac{\partial \mathcal{L}}{\partial \boldsymbol{\zeta}^{\gamma \delta}} \mid \boldsymbol{\psi}\right\rangle & =\frac{r}{|Y|} \eta_{\alpha \beta \gamma \delta}^{B}\left(\boldsymbol{B}_{\alpha \beta \gamma \delta}^{*}-\boldsymbol{B}_{\alpha \beta \gamma \delta}^{\mathrm{target}}\right) \int_{Y} \boldsymbol{C}(\boldsymbol{y}):\left(\boldsymbol{E}^{\gamma \delta}+\boldsymbol{\varepsilon}_{\boldsymbol{y}}\left(\boldsymbol{\xi}^{\gamma \delta}\right)\right): \boldsymbol{\varepsilon}_{\boldsymbol{y}}(\boldsymbol{\psi}) d \boldsymbol{y} \\
& +\frac{r}{|Y|} \eta_{\alpha \beta \gamma \delta}^{D}\left(\boldsymbol{D}_{\alpha \beta \gamma \delta}^{*}-\boldsymbol{D}_{\alpha \beta \gamma \delta}^{\mathrm{target}}\right) \int_{Y} \boldsymbol{C}(\boldsymbol{y}):\left(\boldsymbol{X}^{\gamma \delta}+\boldsymbol{\varepsilon}_{\boldsymbol{y}}\left(\boldsymbol{\zeta}^{\gamma \delta}\right)\right): \boldsymbol{\varepsilon}_{\boldsymbol{y}}(\boldsymbol{\psi}) d \boldsymbol{y} \\
& +\int_{Y} \boldsymbol{C}(\boldsymbol{y}): \boldsymbol{\varepsilon}_{\boldsymbol{y}}(\boldsymbol{\psi}): \boldsymbol{\varepsilon}_{\boldsymbol{y}}(\boldsymbol{Z}) d \boldsymbol{y}
\end{aligned}
$$

The integral over $Y$ on the first two lines is equal to 0 from the state equations (2.7) and (2.8). Moreover, by choosing $\psi=\boldsymbol{Z}$ and using the positive definiteness of $\boldsymbol{C}$ as well as the $Y$-periodicity of $\boldsymbol{Z}$, we obtain that the solution of the adjoint state is identically zero, $\boldsymbol{Z}=\mathbf{0}$.

Shape derivative. Deforming the interface $\Gamma$ in the direction of a smooth vector field $\boldsymbol{\theta}$, the shape derivative of the objective function is found to be the shape derivative of the Lagrangian at the optimal point [34]:

$$
\mathcal{J}^{\prime}(S)(\boldsymbol{\theta})=\left\langle\frac{\partial \mathcal{L}}{\partial S}\left(S, \boldsymbol{w}^{\gamma \delta}, \mathbf{0}, \boldsymbol{p}^{\gamma \delta}, \mathbf{0}\right) \mid \boldsymbol{\theta}\right\rangle
$$

Thus:

$$
\begin{aligned}
\mathcal{J}^{\prime}(S)(\boldsymbol{\theta}) & =\frac{r}{|Y|}\left\|\boldsymbol{A}^{*}\left(d_{S}\right)-\boldsymbol{A}^{\mathrm{target}}\right\|_{\eta_{A}} \int_{Y} d_{S}^{\prime}(\boldsymbol{\theta}) \boldsymbol{C}^{\prime}\left(d_{S}\right):\left(\boldsymbol{E}^{\gamma \delta}+\boldsymbol{\varepsilon}_{\boldsymbol{y}}\left(\boldsymbol{w}^{\gamma \delta}\right)\right):\left(\boldsymbol{E}^{\alpha \beta}+\boldsymbol{\varepsilon}_{\boldsymbol{y}}\left(\boldsymbol{w}^{\alpha \beta}\right)\right) d \boldsymbol{y} \\
& +\frac{r}{|Y|}\left\|\boldsymbol{B}^{*}\left(d_{S}\right)-\boldsymbol{B}^{\mathrm{target}}\right\|_{\eta_{B}} \int_{Y} d_{S}^{\prime}(\boldsymbol{\theta}) \boldsymbol{C}^{\prime}\left(d_{S}\right):\left(\boldsymbol{E}^{\gamma \delta}+\boldsymbol{\varepsilon}_{\boldsymbol{y}}\left(\boldsymbol{w}^{\gamma \delta}\right)\right):\left(\boldsymbol{X}^{\alpha \beta}+\boldsymbol{\varepsilon}_{\boldsymbol{y}}\left(\boldsymbol{p}^{\alpha \beta}\right)\right) d \boldsymbol{y} \\
& +\frac{r}{|Y|}\left\|\boldsymbol{D}^{*}\left(d_{S}\right)-\boldsymbol{D}^{\mathrm{target}}\right\|_{\eta_{D}} \int_{Y} d_{S}^{\prime}(\boldsymbol{\theta}) \boldsymbol{C}^{\prime}\left(d_{S}\right):\left(\boldsymbol{X}^{\gamma \delta}+\boldsymbol{\varepsilon}_{\boldsymbol{y}}\left(\boldsymbol{p}^{\gamma \delta}\right)\right):\left(\boldsymbol{X}^{\alpha \beta}+\boldsymbol{\varepsilon}_{\boldsymbol{y}}\left(\boldsymbol{p}^{\alpha \beta}\right)\right) d \boldsymbol{y}
\end{aligned}
$$

Moreover, using the results presented in Proposition 2.5 and then Proposition 2.9 from [55], the shape derivative from 
above can be rewritten as follows:

$$
\begin{aligned}
\mathcal{J}^{\prime}(S)(\boldsymbol{\theta}) & =\frac{r}{|Y|}\left\|\boldsymbol{A}^{*}\left(d_{S}\right)-\boldsymbol{A}^{\mathrm{target}}\right\|_{\eta_{A}} \int_{\Gamma^{S}}-\boldsymbol{\theta} \cdot \boldsymbol{n} f_{A}(\boldsymbol{s}) d \boldsymbol{s} \\
& +\frac{r}{|Y|}\left\|\boldsymbol{B}^{*}\left(d_{S}\right)-\boldsymbol{B}^{\mathrm{target}}\right\|_{\eta_{B}} \int_{\Gamma^{S}}-\boldsymbol{\theta} \cdot \boldsymbol{n} f_{B}(\boldsymbol{s}) d \boldsymbol{s} \\
& +\frac{r}{|Y|}\left\|\boldsymbol{D}^{*}\left(d_{S}\right)-\boldsymbol{D}^{\mathrm{target}}\right\|_{\eta_{D}} \int_{\Gamma^{S}} \boldsymbol{\theta} \cdot \boldsymbol{n} f_{D}(\boldsymbol{s}) d \boldsymbol{s}
\end{aligned}
$$

where $f_{A}(s), f_{B}(s)$ and $f_{D}(s)$ are scalar fields defined by:

$$
\left\{\begin{array}{l}
f_{A}(\boldsymbol{s})=\int_{\operatorname{ray}_{\Gamma} \cap Y}\left[\prod_{i=1}^{2} 1+d_{S}(\boldsymbol{z}) \kappa_{i}(\boldsymbol{s})\right] \mathcal{H}_{e}^{\prime}\left(d_{S}\right)\left(\boldsymbol{C}^{\bar{S}}-\boldsymbol{C}^{S}\right):\left(\boldsymbol{E}^{\gamma \delta}+\boldsymbol{\varepsilon}_{z}\left(\boldsymbol{w}^{\gamma \delta}\right)\right):\left(\boldsymbol{E}^{\alpha \beta}+\boldsymbol{\varepsilon}_{z}\left(\boldsymbol{w}^{\alpha \beta}\right)\right) d \boldsymbol{z} \\
f_{B}(\boldsymbol{s})=\int_{\operatorname{ray}_{\Gamma} \cap Y}\left[\prod_{i=1}^{2} 1+d_{S}(\boldsymbol{z}) \kappa_{i}(\boldsymbol{s})\right] \mathcal{H}_{e}^{\prime}\left(d_{S}\right)\left(\boldsymbol{C}^{\bar{S}}-\boldsymbol{C}^{S}\right):\left(\boldsymbol{E}^{\gamma \delta}+\boldsymbol{\varepsilon}_{z}\left(\boldsymbol{w}^{\gamma \delta}\right)\right):\left(\boldsymbol{X}^{\alpha \beta}+\boldsymbol{\varepsilon}_{z}\left(\boldsymbol{p}^{\alpha \beta}\right)\right) d \boldsymbol{z} \\
f_{D}(\boldsymbol{s})=\int_{\operatorname{ray}_{\Gamma} \cap \cap}\left[\prod_{i=1}^{2} 1+d_{S}(\boldsymbol{z}) \kappa_{i}(\boldsymbol{s})\right] \mathcal{H}_{e}^{\prime}\left(d_{S}\right)\left(\boldsymbol{C}^{\bar{S}}-\boldsymbol{C}^{S}\right):\left(\boldsymbol{X}^{\gamma \delta}+\boldsymbol{\varepsilon}_{z}\left(\boldsymbol{p}^{\gamma \delta}\right)\right):\left(\boldsymbol{X}^{\alpha \beta}+\boldsymbol{\varepsilon}_{z}\left(\boldsymbol{p}^{\alpha \beta}\right)\right) d \boldsymbol{z}
\end{array}\right.
$$

In the above equation, $\kappa_{i}(s)$ denote the principal curvatures of $\Gamma^{S}$.

Approximate formula for the shape derivative. Although formula (A.3) is satisfying from a mathematical point of view, its numerical evaluation is not completely straightforward. There are two delicate issues. First, one has to compute the principal curvatures $\kappa_{i}(s)$ for any point $s \in \Gamma$ on the interface. Second, one has to perform an integration along the rays of the energy-like quantity. This is a classical task in the level set framework but, still, it is of interest to devise a simpler approximate formula for the shape derivative.

Following the ideas developed in [55, 65], a first approximate formula is to assume that the interface is roughly plane, namely to assume that the principal curvatures $\kappa_{i}(\boldsymbol{s})$ vanish. In such a case we obtain a "Jacobian-free" approximate shape derivative. This gives a new expression for $f_{A}, f_{B}$ and $f_{D}$ :

$$
\left\{\begin{array}{l}
f_{A}(\boldsymbol{s})=\int_{\mathrm{ray}_{\Gamma} \cap Y} \mathcal{H}_{e}^{\prime}\left(d_{S}\right)\left(\boldsymbol{C}^{\bar{S}}-\boldsymbol{C}^{S}\right):\left(\boldsymbol{E}^{\gamma \delta}+\boldsymbol{\varepsilon}_{z}\left(\boldsymbol{w}^{\gamma \delta}\right)\right):\left(\boldsymbol{E}^{\alpha \beta}+\boldsymbol{\varepsilon}_{z}\left(\boldsymbol{w}^{\alpha \beta}\right)\right) d z \\
f_{B}(\boldsymbol{s})=\int_{\operatorname{ray}_{\Gamma^{S}} \cap Y} \mathcal{H}_{e}^{\prime}\left(d_{S}\right)\left(\boldsymbol{C}^{\bar{S}}-\boldsymbol{C}^{S}\right):\left(\boldsymbol{E}^{\gamma \delta}+\boldsymbol{\varepsilon}_{z}\left(\boldsymbol{w}^{\gamma \delta}\right)\right):\left(\boldsymbol{X}^{\alpha \beta}+\boldsymbol{\varepsilon}_{z}\left(\boldsymbol{p}^{\alpha \beta}\right)\right) d z \\
f_{D}(\boldsymbol{s})=\int_{\operatorname{ray}_{\Gamma} \cap Y} \mathcal{H}_{e}^{\prime}\left(d_{S}\right)\left(\boldsymbol{C}^{\bar{S}}-\boldsymbol{C}^{S}\right):\left(\boldsymbol{X}^{\gamma \delta}+\boldsymbol{\varepsilon}_{z}\left(\boldsymbol{p}^{\gamma \delta}\right)\right):\left(\boldsymbol{X}^{\alpha \beta}+\boldsymbol{\varepsilon}_{z}\left(\boldsymbol{p}^{\alpha \beta}\right)\right) d z
\end{array}\right.
$$

A second approximate formula is obtained when the smoothing parameter $e$ is small. Note that, since the support of the function $h_{e}$ is of size $2 e$, the integral in formula (A.3) is confined to a tubular neighbourhood of $\Gamma$ of width $2 e$. Therefore, if $e$ is small, one may assume that the functions depending on $z$ are constant along each ray, equal to their value at $\boldsymbol{y} \in \Gamma$. In other words, for small $e$ we assume:

$$
\varepsilon_{z} \approx \varepsilon_{s} \quad d_{S}(z) \approx d_{S}(s)=0
$$

which yields the approximate formulas, for $\boldsymbol{y} \in \Gamma^{S}$ : 


$$
\left\{\begin{array}{l}
f_{A}(\boldsymbol{s})=\left(\boldsymbol{C}^{\bar{S}}-\boldsymbol{C}^{S}\right):\left(\boldsymbol{E}^{\gamma \delta}+\boldsymbol{\varepsilon}_{\boldsymbol{s}}\left(\boldsymbol{w}^{\gamma \delta}\right)\right):\left(\boldsymbol{E}^{\alpha \beta}+\boldsymbol{\varepsilon}_{\boldsymbol{s}}\left(\boldsymbol{w}^{\alpha \beta}\right)\right) \int_{\mathrm{ray}_{\Gamma} \cap Y} \mathcal{H}_{e}^{\prime}\left(d_{S}\right) d z \\
f_{B}(\boldsymbol{s})=\left(\boldsymbol{C}^{\bar{S}}-\boldsymbol{C}^{S}\right):\left(\boldsymbol{E}^{\gamma \delta}+\boldsymbol{\varepsilon}_{\boldsymbol{s}}\left(\boldsymbol{w}^{\gamma \delta}\right)\right):\left(\boldsymbol{X}^{\alpha \beta}+\boldsymbol{\varepsilon}_{\boldsymbol{s}}\left(\boldsymbol{p}^{\alpha \beta}\right)\right) \int_{\mathrm{ray}_{\mathrm{\Gamma}} \cap Y} \mathcal{H}_{e}^{\prime}\left(d_{S}\right) d z \\
f_{D}(\boldsymbol{s})=\left(\boldsymbol{C}^{\bar{S}}-\boldsymbol{C}^{S}\right):\left(\boldsymbol{X}^{\gamma \delta}+\boldsymbol{\varepsilon}_{\boldsymbol{s}}\left(\boldsymbol{p}^{\gamma \delta}\right)\right):\left(\boldsymbol{X}^{\alpha \beta}+\boldsymbol{\varepsilon}_{\boldsymbol{s}}\left(\boldsymbol{p}^{\alpha \beta}\right)\right) \int_{\mathrm{ray}_{\mathrm{\Gamma}} \cap{ }_{\mathrm{Y}} \cap \mathcal{H}_{e}^{\prime}\left(d_{S}\right) d z}
\end{array}\right.
$$

Furthermore, most rays have a length larger than $2 e$ so that:

$$
\int_{\operatorname{ray}_{\Gamma} \cap \cap Y} \mathcal{H}_{e}^{\prime}\left(d_{\omega}\right) d z+\int_{\operatorname{ray}_{\Gamma} \cap \cap Y} \mathcal{H}_{e}^{\prime}\left(d_{\omega}\right) d z=\mathcal{H}_{e}(e)-\mathcal{H}_{e}(-e)=1 .
$$

In turn, the shape derivative in (A.3) can be approximated by:

$$
\begin{aligned}
\mathcal{J}^{\prime}(S)(\boldsymbol{\theta}) & =\frac{r}{|Y|}\left\|\boldsymbol{A}^{*}\left(d_{S}\right)-\boldsymbol{A}^{\mathrm{target}}\right\|_{\eta_{A}} \int_{\Gamma^{S}}-\boldsymbol{\theta} \cdot \boldsymbol{n}\left(\boldsymbol{C}^{\bar{S}}-\boldsymbol{C}^{S}\right):\left(\boldsymbol{E}^{\gamma \delta}+\boldsymbol{\varepsilon}_{\boldsymbol{s}}\left(\boldsymbol{w}^{\gamma \delta}\right)\right):\left(\boldsymbol{E}^{\alpha \beta}+\boldsymbol{\varepsilon}_{\boldsymbol{s}}\left(\boldsymbol{w}^{\alpha \beta}\right)\right) d \boldsymbol{s} \\
& +\frac{r}{|Y|}\left\|\boldsymbol{B}^{*}\left(d_{S}\right)-\boldsymbol{B}^{\mathrm{target}}\right\|_{\eta_{B}} \int_{\Gamma^{S}} \boldsymbol{\theta} \cdot \boldsymbol{n}\left(\boldsymbol{C}^{\bar{S}}-\boldsymbol{C}^{S}\right):\left(\boldsymbol{E}^{\gamma \delta}+\boldsymbol{\varepsilon}_{\boldsymbol{s}}\left(\boldsymbol{w}^{\gamma \delta}\right)\right):\left(\boldsymbol{X}^{\alpha \beta}+\boldsymbol{\varepsilon}_{\boldsymbol{s}}\left(\boldsymbol{p}^{\alpha \beta}\right)\right) d \boldsymbol{s} \\
& +\frac{r}{|Y|}\left\|\boldsymbol{D}^{*}\left(d_{S}\right)-\boldsymbol{D}^{\mathrm{target}}\right\|_{\eta_{D}} \int_{\Gamma^{S}} \boldsymbol{\theta} \cdot \boldsymbol{n}\left(\boldsymbol{C}^{\bar{S}}-\boldsymbol{C}^{S}\right):\left(\boldsymbol{X}^{\gamma \delta}+\boldsymbol{\varepsilon}_{z}\left(\boldsymbol{p}^{\gamma \delta}\right)\right):\left(\boldsymbol{X}^{\alpha \beta}+\boldsymbol{\varepsilon}_{z}\left(\boldsymbol{p}^{\alpha \beta}\right)\right) d \boldsymbol{s}
\end{aligned}
$$

Numerical results performed in [55] reveal that the latter simplification (A.6), which we shall refer to as the approximate shape derivative, works very well in practice for problems of compliance minimization. Formula (A.6) is also used by Wang et al. in their numerical simulations [42].

\section{Appendix B. Volume constraint}

Two-sided inequality constraint. We recall that the set of admissible shape $\mathcal{U}_{a d}$ for the optimization problem (3.3) is defined in eq. (3.1). The present section discusses the augmented Lagrangian approach, used to prescribe a two-sided inequality constraints. The discussion is included here only for completeness, is originally presented in the book of Bertsekas [63] (refer also to Chapter 17 of the book by Nocedal and Wright [64]).

The optimization problem (3.3) can be traded with a constraint-free minimization of a (Lagrangian-like) weighted sum of the cost functional $\mathcal{J}(S)$ and the constraint $\mathcal{P}(S, \lambda, \mu)$ that reads:

$$
\begin{aligned}
& \inf (\mathcal{J}(S)+\mathcal{P}(S, \lambda, \mu)) \\
& \mathcal{P}(S, \lambda, \mu)=\min _{\rho_{m} \leq|S|-v \leq \rho_{M}}\left(\lambda v+\frac{\mu}{2}|v|^{2}\right)
\end{aligned}
$$

where $v$ is a slack variable, $\lambda$ is the Lagrange multiplier for the volume constraint and $\mu$ is a penalty paramater. At iteration $k$, a straightforward calculation shows that the minimum above is attained at the point $v^{k}$ given by:

$$
v^{k}= \begin{cases}|S|-\rho_{M} & \text { if } \lambda^{k}+\mu^{k}\left(|S|-\rho_{M}\right)>0 \\ |S|-\rho_{m} & \text { if } \lambda^{k}+\mu^{k}\left(|S|-\rho_{m}\right)<0 \\ -\lambda^{k} / \mu^{k} & \text { otherwise }\end{cases}
$$


and $\mathcal{P}(S, \lambda, \mu)$ is given by:

$$
\mathcal{P}(S, \lambda, \mu)= \begin{cases}\lambda^{k}\left(|S|-\rho_{M}\right)+\frac{\mu^{k}}{2}|| S\left|-\rho_{M}\right|^{2} & \text { if } \lambda^{k}+\mu^{k}\left(|S|-\rho_{M}\right)>0 \\ \lambda^{k}\left(|S|-\rho_{m}\right)+\frac{\mu^{k}}{2}|| S\left|-\rho_{m}\right|^{2} & \text { if } \lambda^{k}+\mu^{k}\left(|S|-\rho_{m}\right)<0 \\ -\left(\lambda^{k}\right)^{2} / 2 \mu^{k} & \text { otherwise }\end{cases}
$$

The conclusion from the preceding analysis is that a method of multipliers for problem consists of sequential minimizations of the form, which do not involve the variable $v$. The (first-order) multiplier iteration is given by:

$$
\lambda_{k+1}= \begin{cases}\lambda^{k}+\mu^{k}\left(|S|-\rho_{M}\right) & \text { if } \lambda^{k}+\mu^{k}\left(|S|-\rho_{M}\right)>0 \\ \lambda^{k}+\mu^{k}\left(|S|-\rho_{m}\right) & \text { if } \lambda^{k}+\mu^{k}\left(|S|-\rho_{m}\right)<0 \\ 0 & \text { otherwise }\end{cases}
$$

The last aspect consists in updating the penalty parameters $\mu$ every 10 iterations as follows:

$$
\mu^{k+10}=2 \mu^{k}
$$

Shape sensitivity analysis. Deforming the interface $\Gamma^{S}$ in the direction of a smooth vector field $\boldsymbol{\theta}$, the shape derivative of the constraint function $\mathcal{P}(S, \lambda, \mu)$ under the approximation of thin smooth interphase is obtained by analogy with the results in the previous appendix:

$$
\mathcal{P}^{\prime}(S, \lambda, \mu)(\boldsymbol{\theta})= \begin{cases}{\left[\lambda^{k}+\left(|S|-\rho_{M}\right) \mu^{k}\right] \int_{\Gamma_{S}}-\boldsymbol{\theta} \cdot \boldsymbol{n} d \boldsymbol{s}} & \text { if } \lambda^{k}+\mu^{k}\left(|S|-\rho_{M}\right)>0 \\ {\left[\lambda^{k}+\left(|S|-\rho_{m}\right) \mu^{k}\right] \int_{\Gamma_{S}}^{-\boldsymbol{\theta} \cdot \boldsymbol{n} d \boldsymbol{s}}} & \text { if } \lambda^{k}+\mu^{k}\left(|S|-\rho_{m}\right)<0 \\ 0 & \text { otherwise }\end{cases}
$$

\section{Appendix C. Constitutive behavior of laminate plate as a route for prescribing targets}

The choice of a target plate tensor may seem a difficult task a priori. The prescribed stiffness coefficients should not compromise the positive definiteness, and should remain bounded imposed by the rule of mixture (e.g. the VoigtReuss bounds). Yet, to the best of our knowledge, the clear definition of elastic bounds in the context of thin plates has not been explored, and is beyond the scope of the present work. We rather address this aspect by studying the laminate plate, a sub-category of elastic plate with periodic pattern. This simpler framework permits to rapidly construct achievable target tensors analytically. In the sequel, we recall the expressions of $\boldsymbol{A}, \boldsymbol{B}, \boldsymbol{D}$ in the context of the classical laminate plate theory (CPLT) [31], and illustrate the construction of a target through an simple case which is used in the numerical examples (section 5).

Note that a limiting case for a homogeneous thin plate theory should be the Kirchhoff-Love plate equations. Let us consider the definition of $\boldsymbol{N}$ as a sum of integrals in each layer:

$$
\boldsymbol{N}=\sum_{k=1}^{n} \int_{h_{k}}^{h_{k+1}} \boldsymbol{\sigma} d x_{3}
$$


Introducing the constitutive behavior layer by layer and using the generalised strain components gives:

$$
\boldsymbol{N}=\sum_{k=1}^{n} \int_{h_{k}}^{h_{k+1}} \boldsymbol{C}:\left(\boldsymbol{\varepsilon}(\boldsymbol{U})+x_{3} \chi\left(U_{3}\right)\right) d x_{3}
$$

Since the generalised strains do not depend upon $x_{3}$, one can write:

$$
\begin{aligned}
\boldsymbol{N} & =\left[\sum_{k=1}^{n} \int_{h_{k}}^{h_{k+1}} \boldsymbol{C} d x_{3}\right]: \boldsymbol{\varepsilon}(\boldsymbol{U})+\left[\sum_{k=1}^{n} \int_{h_{k}}^{h_{k+1}} x_{3} \boldsymbol{C} d x_{3}\right]: \chi\left(U_{3}\right) \\
& =\boldsymbol{A}: \boldsymbol{\varepsilon}(\boldsymbol{U})+\boldsymbol{B}: \boldsymbol{\chi}\left(U_{3}\right)
\end{aligned}
$$

Following the same reasoning for $\mathrm{M}$ we can write:

$$
\begin{aligned}
\boldsymbol{M} & =\left[\sum_{k=1}^{n} \int_{h_{k}}^{h_{k+1}} x_{3} \boldsymbol{C} d x_{3}\right]: \boldsymbol{\varepsilon}(\boldsymbol{U})+\left[\sum_{k=1}^{n} \int_{h_{k}}^{h_{k+1}} x_{3}^{2} \boldsymbol{C} d x_{3}\right]: \chi\left(U_{3}\right) \\
& =\boldsymbol{B}: \boldsymbol{\varepsilon}(\boldsymbol{U})+\boldsymbol{D}: \chi\left(U_{3}\right)
\end{aligned}
$$

Thus, the plate constitutive law is:

$$
\left[\begin{array}{l}
N \\
M
\end{array}\right]=\left[\begin{array}{ll}
A & B \\
B & D
\end{array}\right]:\left[\begin{array}{l}
\varepsilon \\
\chi
\end{array}\right]
$$

Like in the thin plates with periodic microstructure, the general laminate plate model induces an extension-bending coupling in the most general case. It is a consequence of the heterogeneous or anisotropic properties of the panel (variations between each ply). To illustrate this effect, let us consider a simple bi-phase composite panels, i.e. composed by two superposed plates that are perfectly glued at their interface. It is assumed in this example that the upper plate is stiffer than the lower one in the direction $\left(O, \boldsymbol{e}_{1}\right)$. Under a tensile loading in the direction $\left(O, \boldsymbol{e}_{1}\right)$, not only the plate is stretched in the direction $\left(O, \boldsymbol{e}_{1}\right)$, it also undergoes an out of plane curvature (hence a coupled response).

The main difference between the laminate plate theory and the panel with periodic microstructure lies in the fact that $\boldsymbol{B}$ is symmetric in the case of laminates, but not necessarily in the case of periodic plates.

Example. Let us consider a bi-phase laminate plate of thickness $2 h$, composed of isotropic plies of equal thickness. The material in the upper ply $S_{p}$ is described by Young's modulus $E_{p}=0.4608 \mathrm{MPa}$ and Poisson's ratio is $v_{p}=-0.2$, whereas the material in lower ply $S_{m}$ features a Young's modulus $E_{m}=0.1728 \mathrm{MPa}$ and Poisson's ratio is $v_{m}=-0.8$. The resulting laminate plate stiffness tensor, computed analytically from eqs. (C.1) and (C.2), reads:

$$
\boldsymbol{C}^{*}=\left[\begin{array}{ccc|ccc}
0.12 & -0.06 & 0 & 0 & 2.3 \mathrm{e}^{-3} & 0 \\
-0.06 & 0.12 & 0 & 2.3 \mathrm{e}^{-3} & 0 & 0 \\
0 & 0 & 0.09 & 0 & 0 & -1.1 \mathrm{e}^{-3} \\
\hline 0 & 2.3 \mathrm{e}^{-3} & 0 & 6.3 \mathrm{e}^{-4} & -3.1 \mathrm{e}^{-4} & 0 \\
2.3 \mathrm{e}^{-3} & 0 & 0 & -3.1 \mathrm{e}^{-4} & 6.3 \mathrm{e}^{-4} & 0 \\
0 & 0 & -1.1 \mathrm{e}^{-3} & 0 & 0 & 4.7 \mathrm{e}^{-4}
\end{array}\right]
$$


The compliance tensor $\boldsymbol{S}^{*}$, which is obtained by taking the inverse of $\boldsymbol{C}^{*}$, gives a better understanding of the plate kinematics, because for a given (simple) input stress field, we can see all the deformation that are triggered:

$$
\boldsymbol{S}^{*}=\left[\begin{array}{ccc|ccc}
14.28 & 8.55 & 0 & -75.32 & -89.06 & 0 \\
8.55 & 14.28 & 0 & -89.06 & -75.32 & 0 \\
0 & 0 & 0.09 & 0 & 0 & 27.49 \\
\hline-75.32 & -89.06 & 0 & 2742 & 1642 & 0 \\
-89.06 & -75.32 & 0 & 1642 & 2742 & 0 \\
0 & 0 & 27.49 & 0 & 0 & 2199
\end{array}\right]
$$

The process can be extended to laminate with $n$ ply, where each ply is orthotropic.

\section{Appendix D. Compliance tensors}

Appendix D.1. Example 1

$$
\boldsymbol{S}^{*}=\left[\begin{array}{ccc|ccc}
11.83 & 5.74 & 0 & 11.23 & -35.85 & 0 \\
5.74 & 11.54 & 0 & -19.10 & -20.15 & 0 \\
0 & 0 & 33.82 & 0 & 0 & 53.71 \\
\hline 11.23 & -19.10 & 0 & 1723 & 61.42 & 0 \\
-35.85 & -20.15 & 0 & 61.42 & 1723 & 0 \\
0 & 0 & 53.71 & 0 & 0 & 5968
\end{array}\right]
$$

Appendix D.2. Example 2

$$
\boldsymbol{S}^{*}=\left[\begin{array}{ccc|ccc}
11.74 & 4.02 & 0 & -14.23 & -10.47 & 0 \\
4.02 & 11.63 & 0 & -13.12 & -12.33 & 0 \\
0 & 0 & 43.79 & 0 & 0 & -39.41 \\
\hline-14.23 & -13.12 & 0 & 3934 & -878.1 & 0 \\
-10.47 & -12.33 & 0 & -878.1 & 3927 & 0 \\
0 & 0 & -39.41 & 0 & 0 & 5035
\end{array}\right]
$$

Appendix D.3. Example 3

$$
\boldsymbol{S}^{*}=\left[\begin{array}{ccc|ccc}
12.20 & 5.73 & 0 & -49.99 & -15.15 & 0 \\
5.73 & 11.33 & 0 & -19.07 & -34.12 & 0 \\
0 & 0 & 38.85 & 0 & 0 & -103.2 \\
\hline-49.99 & -19.07 & 0 & 1352 & -45.51 & 0 \\
-15.15 & -34.12 & 0 & -45.51 & 1175 & 0 \\
0 & 0 & -103.2 & 0 & 0 & 3399
\end{array}\right]
$$


Appendix D.4. Example 4

$$
\boldsymbol{S}^{*}=\left[\begin{array}{ccc|ccc}
100.2 & 0.27 & 0 & -1013 & -11.37 & 0 \\
0.27 & 28.70 & 0 & -7.794 & 299.0 & 0 \\
0 & 0 & 136.8 & 0 & 0 & -124.4 \\
\hline-1013 & -7.794 & 0 & 11302 & -203.0 & 0 \\
-15.15 & -34.12 & 0 & -203.0 & 4486 & 0 \\
0 & 0 & -124.4 & 0 & 0 & 9204
\end{array}\right]
$$

